\title{
De la recherche à la conception programmatique et à la mise en oeuvre : Programmation à l'intention des filles des milieux ruraux d'Éthiopie-Boîte à outils du praticien
}

Annabel Erulkar

Population Council

Follow this and additional works at: https://knowledgecommons.popcouncil.org/departments_sbsr-pgy

Part of the Demography, Population, and Ecology Commons, Family, Life Course, and Society Commons, Gender and Sexuality Commons, International Public Health Commons, Medicine and Health Commons, and the Rural Sociology Commons

How does access to this work benefit you? Let us know!

\section{Recommended Citation}

Erulkar, Annabel. 2012. "De la recherche à la conception programmatique et à la mise en oeuvre :

Programmation à l'intention des filles des milieux ruraux d'Éthiopie-Boîte à outils du praticien." Addis Ababa: Population Council. 


\section{DE LA RECHERCHE À LA CONCEPTION PROGRAMMATIQUE ET À LA MISE EN CEUVRE PROGRAMMATION À L'INTENTION DES FILLES
DES MILIEUX RURAUX D'ÉTHIOPIE}

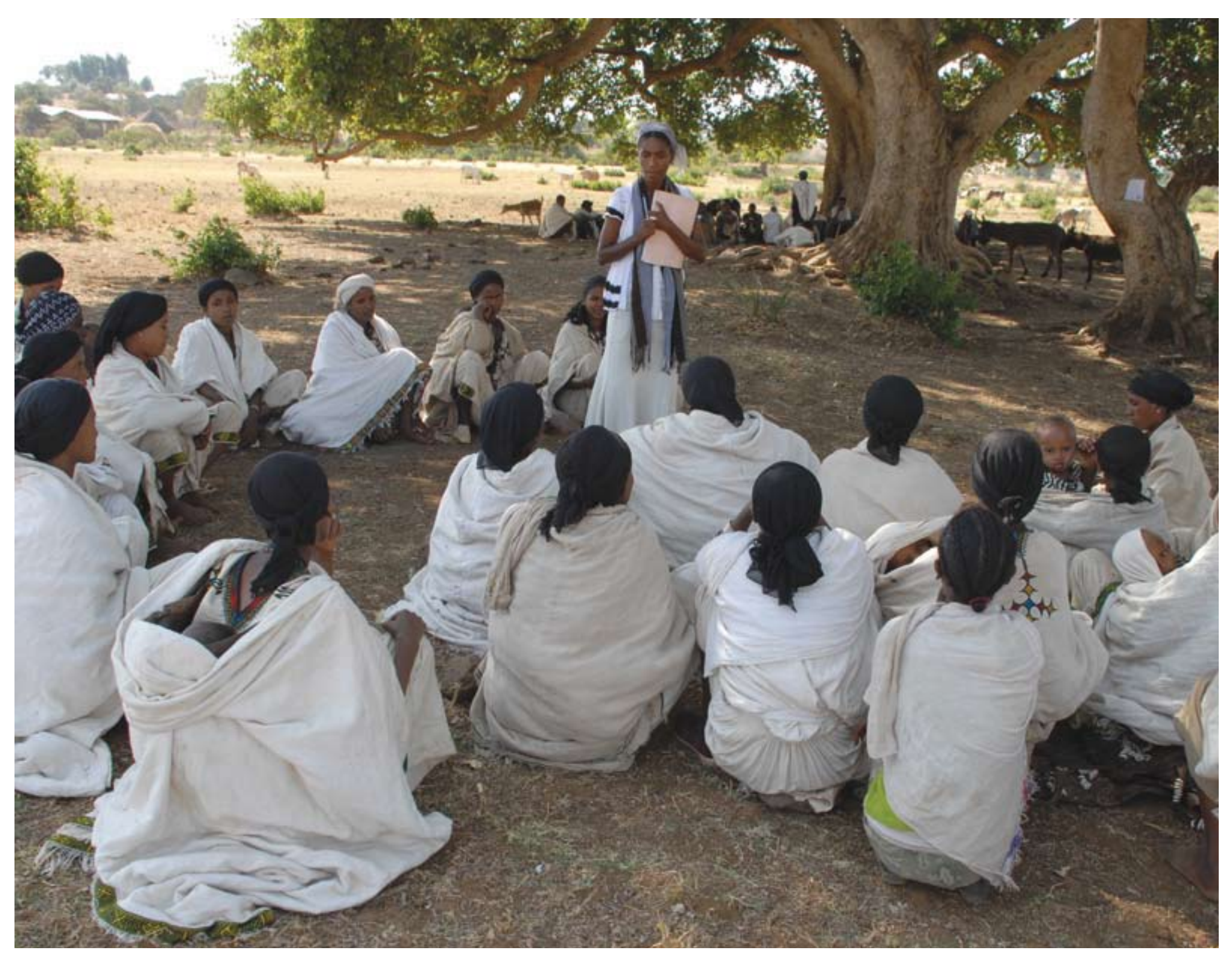




\section{(1) Population Council}

Le Population Council s'attelle aux questions cruciales de santé et de développement - qu'il s'agisse d'endiguer la propagation du VIH, d'améliorer la santé reproductive et d'assurer que les jeunes mènent une vie satisfaisante et productive. Grâce à des travaux de recherche en biomédecine, en sciences sociales et en santé publique dans 50 pays, le Population Council travaille avec ses partenaires pour offrir des solutions qui conduisent à des politiques, des programmes et des technologies plus efficaces pour améliorer la vie partout dans le monde. Fondé en 1952 et basé à New York, le Population Council est une organisation non gouvernementale à but non lucratif avec un conseil d'administration international.

(C) 2012 The Population Council, Inc.

Population Council

P.O. 25562, Code 1000

Addis Abeba, ÉTHIOPIE

Tél.: +(251) (0) 116-631-712/4/6

Fax : +(251) (0) 116-631-720

Population Council

One Dag Hammarskjold Plaza

New York, NY 10017 USA

Tél.: +1-212-339-0500

Fax : 212-755-6052

http://www.popcouncil.org

The Nike Foundation, Nike, Inc. et NoVo Foundation

La Nike Foundation (www.nikefoundation.org) investit exclusivement dans les adolescentes, en qui elle voit la force ultime du changement et du soulagement de la pauvreté dans le monde en développement. Ses investissements sont conçus pour mettre les filles à l'ordre du jour des débats du monde et leur assurer les ressources dont elles ont besoin. Les activités de la fondation sont soutenues par la société Nike, Inc. et par la NoVo Foundation, dont l'effort de collaboration a intensifié considérablement l'impact de l'initiative Girl Effect.

Photos : Zeleman Production

Indication de source suggérée : Erulkar A. 2011. " De la recherche à la conception programmatique et à la mise en œuvre : Programmation à l'intention des filles des milieux ruraux d'Éthiopie ", Population Council : Addis Abeba, Éthiopie, mars. 


\section{TABLE DES MATIÈRES}

INTRODUCTION POURQUOI CETTE BOÎTE À OUTILS ? ......................................... i

CHAPITRE 1 RECHERCHE : IDENTIFIER ET DÉCRIRE

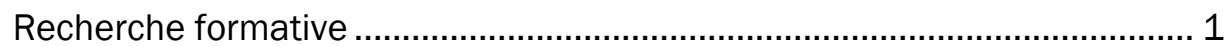

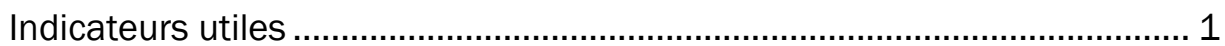

Sources d'information : données primaires et secondaires.......................... 2

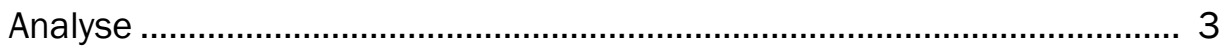

Identification des catégories de filles dans les milieux ruraux:

ce que nous disent les données............................................................... 5

Utiliser les données pour comprendre votre groupe cible............................. 6

OUTILS

1.1 Outil quantitatif de collecte de recherche formative sur les filles

1.2 Outil qualitatif de collecte de recherche formative sur les filles

CHAPITRE 2 CONCEPTION PROGRAMMATIQUE : FAÇONNER L'APPROCHE

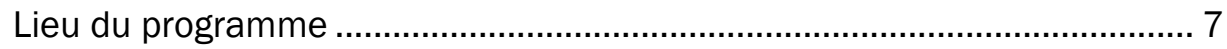

Stratégie / Mode de prestation ................................................................... 9

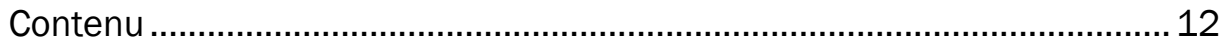

Principes de la programmation................................................................. 14

\section{CHAPITRE 3 MISE EN CEUVRE : RESTER À L'ÉCOUTE DE LA SITUATION SUR LE} TERRAIN ET PRENDRE DU RECUL POUR JUGER L'ENSEMBLE

Rester à l'écoute sur le terrain ................................................................ 17

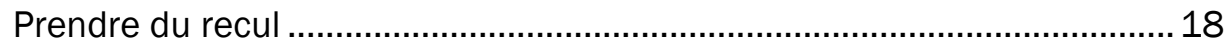

Analyse des données aux fins de la gestion programmatique .................... 19

Remarque concernant la qualité des données statistiques de service......22

Remarque finale sur les programmes factuels ........................................ 22

\section{OUTILS}

3.1 Outil qualitatif de collecte du feedback des filles sur le programme

3.2 Formulaire d'inscription à un programme destiné aux filles

3.3 Formulaire d'activités de programme destiné aux filles 


\begin{abstract}
et ouvrage a été rédigé par Annabel Erulkar et repose sur l'expérience du Population Council en matière de conception de programmes destinés aux filles d'Afrique subsaharienne. L'auteur tient à remercier les membres du Brain Trust of Practitioners d'Éthiopie pour avoir mis en lumière les besoins des filles des milieux ruraux et porté à l'avant-plan les difficultés de la programmation les concernant. Merci à Aster Tefera et Kelemua Hailemariam pour la coordination du Brain Trust et pour leurs contributions à cette "boîte à outils ". À Herma Gebru pour la compilation des ressources relatives aux filles publiée ici et à Eunice Muthengi pour son assistance à l'ébauche des versions antérieures de l'ouvrage. À Wendy Baldwin, Amy Babcheck et Judith Bruce pour leurs très précieux commentaires sur les versions antérieures. Merci enfin à la Nike Foundation pour son soutien au Brain Trust et au développement de cette boîte à outils, ainsi que pour son engagement continu envers les filles des milieux ruraux du monde en développement. En particulier, merci à Amy Babcheck pour la constance de son soutien et de son encouragement.
\end{abstract}




\section{INTRODUCTION \\ POURQUOI CETTE BOÎTE À OUTILS ?}

$\mathrm{L}$ e dernier recensement éthiopien dénombre plus de 9,3 millions d'adolescentes âgées de 10 à 19 ans. ${ }^{1}$ Comparable aux populations entières de certains pays, ce nombre d'adolescentes en Éthiopie dépasse notamment la population du Bénin (8,9 millions d'habitants), du Burundi (8,3 millions) et de la Suisse (7,7 millions).

La majorité de ces filles (plus de 7,5 millions) vit dans les milieux ruraux de l'Éthiopie. Les filles des milieux ruraux sont particulièrement défavorisées. Par rapport à leurs homologues de sexe masculin et aux jeunes des milieux urbains, elles sont moins susceptibles d'être scolarisées et celles qui le sont bénéficient de moins d'années d'école. Par rapport aux autres jeunes d'Éthiopie, les filles des milieux ruraux sont plus vulnérables aux mariages arrangés; elles se marient plus jeunes et connaissent souvent de premières maternités précoces et non désirées. Elles sont moins susceptibles d'avoir un extrait d'acte de naissance, de participer à la vie civique et de bénéficier des programmes sociaux, mais plus vulnérables aux longues heures de travaux domestiques non rémunérés.

Atteindre les filles des milieux ruraux d'Éthiopie pose de véritables défis. L'infrastructure du pays est extrêmement faible, ce qui veut dire qu'il n'existe guère d'institutions ni de réseaux d'accès à ces filles. Ainsi, si les programmes scolaires offrent généralement un mode d'accès aux jeunes, l'Éthiopie ne compte que 23000 écoles primaires et 1078 écoles secondaires organisées par l'état. ${ }^{2}$ De même, alors que la radio offre un moyen populaire de dissémination de l'information et des messages, l'Éthiopie se caractérise par une faible couverture radiophonique, par rapport même aux autres pays d'Afrique. On estime à 195 le nombre de postes de radio par millier d'habitants en Éthiopie. Par comparaison, en République démocratique du Congo, autre vaste pays rural d'Afrique, ce nombre est estimé à 375 pour mille. Aux États-Unis, il est de 2146 pour mille. ${ }^{3}$

Les zones rurales d'Éthiopie sont du reste physiquement plus difficiles d'accès que celles de la plupart des autres pays. Selon les estimations de la Banque mondiale, l'Éthiopie présente la plus faible densité routière ${ }^{4}$ au monde et $13 \%$ des routes seulement sont revêtues. Le pays ne compte par ailleurs qu'une ligne de chemin de fer, longue de 650 kilomètres à peine. ${ }^{5}$ Cette médiocrité de l'infrastructure et des communications empêche l'accès fondamental à de nombreuses zones rurales où résident les filles, tandis que la propriété limitée de radios réduit davantage encore l'exposition des habitants des milieux ruraux au monde extérieur, au-delà des familles et des communautés proches.

1 Federal Democratic Republic of Ethiopia, Population Census Commission, "Summary and Statistical Report of the 2007 Population and Housing Census," Addis Abeba: Central Statistical Agency, 2008.

2 Ministère de l'Éducation d'Éthiopie (MOE), “Education Statistics Annual Abstract 2000 E.C., 2007-8” Addis Abeba, 2008.

3 Banque mondiale. “World Development Indicators," Washington DC: The World Bank, 2000.

4 Définie comme le rapport entre le nombre de kilomètres de route et la surface en kilomètres carrés du pays.

5 World Bank, lbid. 


\section{Cette " boîte à} outills " décrit un processus d'élaboration programmatique fermement ancré dlans les failts.
"De la recherche à la conception programmatique et à la mise en œuvre: Programmation à l'intention des filles des milieux ruraux d'Éthiopie " propose une boîte outils destinée à l'usage des praticiens d'Éthiopie. L'ouvrage s'adresse à ceux et à celles qui désirent définir ou améliorer les programmes destinés aux adolescentes des milieux ruraux d'Éthiopie. II vise spécifiquement les circonstances spéciales des filles de l'Éthiopie rurale, en vue d'accroître le nombre de programmes organisés à leur intention et de les rendre plus pertinents et mieux adaptés à leur contexte.

Cette " boîte à outils " décrit un processus d'élaboration programmatique fermement ancré dans les faits. Les chapitres de cet ouvrage procèdent dès lors d'un point de départ axé sur la recherche pour identifier et décrire les filles, pour se concentrer ensuite sur le développement programmatique émanant des données et aboutir enfin sur la mise en œuvre guidée par le suivi et l'évaluation permanents. La boîte s'organise en trois chapitres :

Chapitre 1 : RECHERCHE : Identifier et décrire. Ce chapitre met l'accent sur les facteurs indispensables à la compréhension avant la conception de programmes destinés aux filles des milieux ruraux, afin d'assurer l'élaboration de programmes qui relèvent des faits plutôt que de suppositions ou de conjectures. Nous proposons quelques indicateurs fondamentaux, souvent démographiques, utiles à l'identification des sous-groupes de filles et à la description de leurs circonstances. Deux exemples d'outils (l'un quantitatif, l'autre qualitatif) sont inclus pour faciliter la collecte des données d'identification et de description des filles.

Chapitre 2 : CONCEPTION PROGRAMMATIQUE : Façonner l'approche. Ce chapitre définit les grandes lignes d'une approche de conception des programmes destinés aux filles. Les étapes en sont l'identification d'un lieu ou la détermination de la nécessité d'en créer un, l'élaboration de la stratégie ou de la méthode d'information ou de prestation et la suggestion de contenu. Toutes reposent sur les données obtenues des filles. Nous présentons en outre ici les principes guides du développement programmatique.

Chapitre 3 : MISE EN CEUVRE : Rester à l'écoute de la situation sur le terrain et prendre du recul pour juger l'ensemble. Le dernier chapitre propose quelques méthodes de suivi de la mise en œuvre des programmes fondées à la fois sur le suivi étroit des perceptions des bénéficiaires et des intéressés au moyen de techniques qualitatives et sur un examen plus large des tendances d'utilisation.

Tout au long de cet ouvrage, nous nous efforçons de garder nos recommandations simples, pratiques et réalisables. Plutôt que de dresser de longues listes d'information exhaustive, nous préférons distiller les recommandations ou caractéristiques les plus importantes nécessaires à la promotion de programmes de conception factuelle pour les filles d'Éthiopie. Nos recommandations ne sont par conséquent pas nécessairement complètes, mais elles représentent de bonnes pratiques fondamentales de conception programmatique dans notre contexte. Notre espoir est que les responsables programmatiques y trouveront des idées et des outils utiles à l'amélioration et à l'expansion des programmes destinés aux filles d'Éthiopie. 


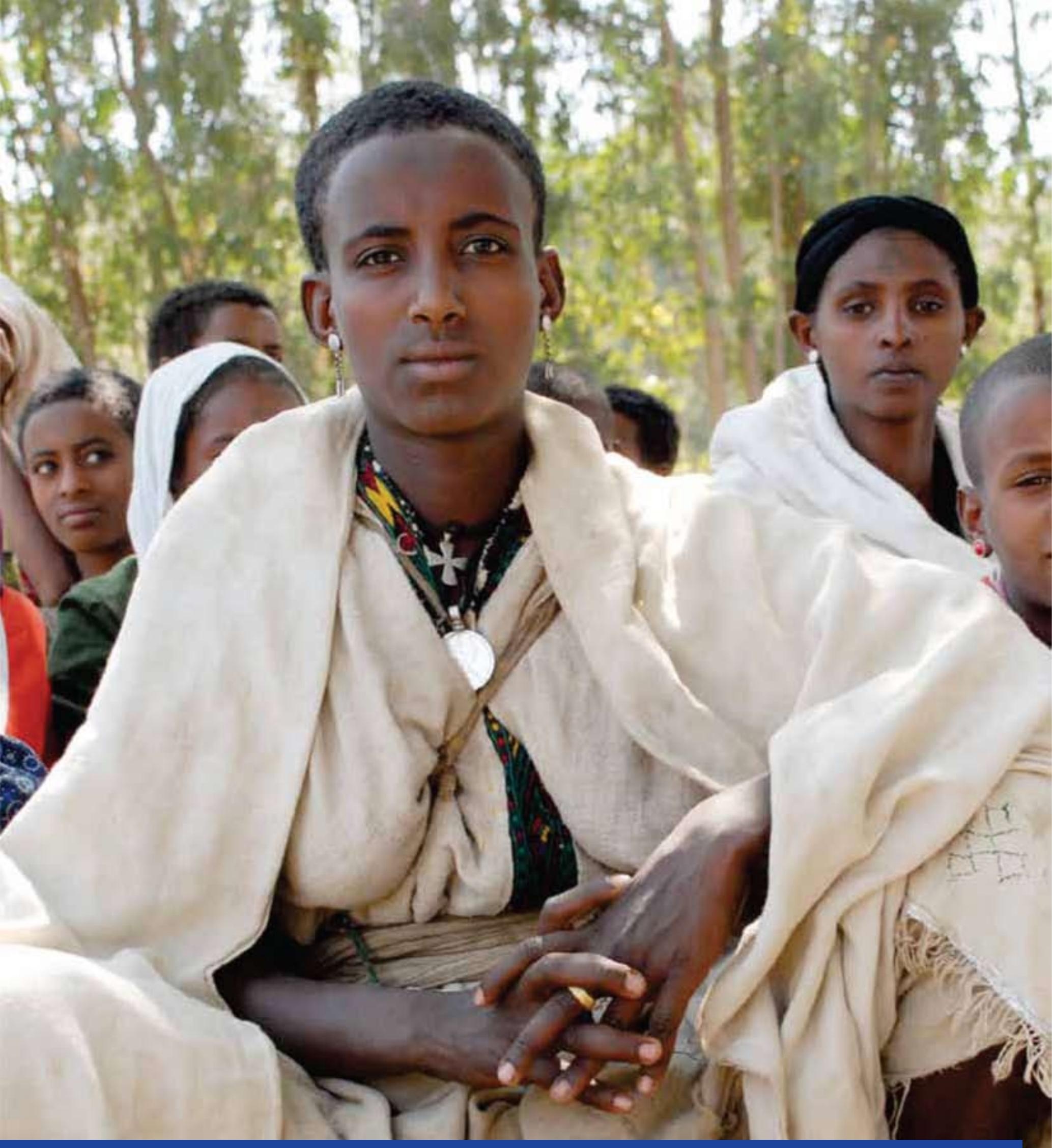

CHAPITRE 1

RECHERCHE: IDENTIFIER ET DÉCRIRE 



\section{CHAPITRE 1 \\ RECHERCHE: IDENTIFIER ET DÉCRIRE}

es programmes pour filles les plus pertinents, les plus efficaces et les plus fructueux sont généralement ceux ancrés dans la réalité des filles elles-mêmes et inspirés d'une base factuelle centrée sur les filles. Un programme véritablement factuel a pour point de départ une image descriptive de la fille.

\section{Recherche formative}

La recherche formative est une recherche menée durant la phase d'élaboration programmatique, pour guider la conception. Avant la phase conceptuelle d'un projet, cette recherche aide les gestionnaires à identifier les catégories de filles ou la problématique de vie des filles en mal d'assistance, ainsi que les aspects à considérer lors de la conception des programmes. Ainsi la recherche formative peut-elle servir de premier bloc de structure au développement programmatique. Si le développement programmatique factuel exige une certaine compétence de la recherche, les organisations disposent de plus en plus de capacités internes de recherche, suivi et évaluation.

Dans cette section, nous examinons l'usage des données pour identifier les catégories de filles à considérer lors du développement des programmes. Les données peuvent aussi servir à décrire la situation des filles visées par le programme et accroître ainsi la pertinence et l'efficacité de ses stratégies. Que vous identifiiez les catégories de filles visées ou que vous essayiez de comprendre leurs circonstances, certains indicateurs clés, ou éléments d'information concernant les filles, sont utiles au processus de conception programmatique.

\section{Indicateurs utiles}

Nombreux sont certes les aspects de la vie des filles dont il faut tenir compte, mais nous recommandons de se concentrer sur quelques traits fondamentaux qui produisent l'information la plus utile à la conception des programmes. Le Tableau 1 présente les caractéristiques clés de la vie des filles et les indicateurs correspondants utiles à l'élaboration de programmes opportuns en ce qu'ils aident à identifier les groupes de filles ou à décrire leurs circonstances. La liste fait principalement état d'indicateurs extraits de sources de données quantitatives (généralement des enquêtes). On remarquera qu'elle peut être modifiée, étendue ou réduite suivant les intérêts poursuivis et la disponibilité des données.

Certains indicateurs peuvent toucher à certaines sensibilités et être source d'embarras pour la fille interviewée, l'intervieweuse ou les deux. II peut par exemple paraître inapproprié dans certains contextes ou circonstances d'interroger les très jeunes filles sur leur expérience sexuelle. II est dans ce cas conseillé de pré-tester les questions douteuses auprès

Un programme véritablement factuel a pour point de départ une image descriptive de la fille. 
TABLEAU 1 Aspects fondamentaux de la vie des filles et indicateurs correspondants utiles à l'élaboration d'un programme

\begin{tabular}{|c|c|}
\hline ASPECTS FONDAMENTAUX & INDICATEURS CLÉS \\
\hline Citoyenneté et enregistrement & $\begin{array}{l}\text { - Pourcentage des filles dont la naissance a été enregistrée } \\
\text { - Pourcentage des filles disposant d'une carte d'identité (de type quelconque) }\end{array}$ \\
\hline Famille et logement & $\begin{array}{l}\text { - Pourcentage vivant avec les deux parents, avec un parent ou sans parent } \\
\text { - Pourcentage d'orphelines } \\
\text { - Pourcentage sous autres dispositions de logement (avec le mari, la belle- } \\
\text { famille, l'employeur, etc.) }\end{array}$ \\
\hline Scolarisation & $\begin{array}{l}\text { - Pourcentage de filles scolarisées } \\
\text { - Pourcentage ayant commencé à fréquenter l'école à l'âge approprié ( } 7 \text { ans) } \\
\text { - Moyenne d'années de scolarité } \\
\text { - Pourcentage ayant accompli un cycle de scolarité (primaire ou secondaire) }\end{array}$ \\
\hline $\begin{array}{l}\text { Réseaux sociaux, participation } \\
\text { et utilisation du temps }\end{array}$ & $\begin{array}{l}\text { - Pourcentage ayant des amies / sans amies } \\
\text { - Pourcentage ayant eu des rapports sociaux durant la dernière semaine, le } \\
\text { dernier mois } \\
\text { - Pourcentage des filles membres d'un club ou d'un groupe } \\
\text { - Pourcentage ayant rendu visite à une pair éducatrice ou s'étant rendues } \\
\text { dans un centre de jeunesse, un établissement de santé ou un } \\
\text { établissement de culte durant le dernier mois } \\
\text { - Moyenne d'heures passées à l'école, au travail rémunéré, au travail non } \\
\text { rémunéré, aux activités sociales et de récréation, au repos }\end{array}$ \\
\hline $\begin{array}{l}\text { Travail rémunéré et non } \\
\text { rémunéré }\end{array}$ & $\begin{array}{l}\text { - Pourcentage ayant travaillé moyennant rémunération } \\
\text { - Type de travail rémunéré, heures et salaire } \\
\text { - Moyenne d'heures passées aux travaux domestiques ou agricoles non } \\
\text { rémunérés }\end{array}$ \\
\hline Activité sexuelle & $\begin{array}{l}\text { - Pourcentage de filles sexuellement expérimentées } \\
\text { - Pourcentage ayant eu des rapports sexuels non consensuels } \\
\text { - Pourcentage utilisant le préservatif et/ou d'autres méthodes de } \\
\text { planification familiale }\end{array}$ \\
\hline Partenariat, mariage & $\begin{array}{l}\text { - Pourcentage des filles ayant déjà été mariées } \\
\text { - Âge moyen au mariage et pourcentage des filles mariées avant l'âge de } 15 \\
\text { et de } 18 \text { ans } \\
\text { - Pourcentage des filles soumises à un mariage arrangé } \\
\text { - Différence d'âge par rapport au mari }\end{array}$ \\
\hline Maternité & $\begin{array}{l}\text { - Pourcentage ayant pratiqué ou désirant pratiquer la planification familiale } \\
\text { - Pourcentage ayant déjà accouché } \\
\text { - Pourcentage ayant reçu des soins de maternité } \\
\text { - Pourcentage ayant bénéficié d'une assistance qualifiée à l'accouchement }\end{array}$ \\
\hline
\end{tabular}

d'un petit échantillon de filles et d'observer leur réaction. II serait par contre peu judicieux d'arrêter soi-même la décision, sans données factuelles ni compréhension de la manière dont les filles réagissent à ces questions dans la pratique. Trop souvent, nous tendons à supposer l'acceptabilité des questions, sans vraiment comprendre comment les filles y réagissent elles-mêmes.

\section{Sources d'information: données primaires et secondaires}

Les sources d'information peuvent inclure des données primaires (celles qu'on collecte soi-même) ou secondaires (celles collectées par autrui). La nouvelle analyse des données secondaires est extrêmement rentable, 
d'autant plus qu'elle évite les coûts de la collecte. Les données collectées à d'autres fins ne couvrent cependant pas nécessairement les variables recherchées ou peuvent avoir été obtenues en dehors de la zone d'intérêt géographique. Measure/DHS [www.measuredhs.com] (pour les Enquêtes démographiques et de santé) et l'International Food Policy Research Institute (IFPRI - Institut international de recherche sur les politiques alimentaires) [www.ifpri.org] offrent des sources courantes de données secondaires accessibles au public et téléchargeables depuis leurs sites Internet. D'autres institutions de recherche et universités locales peuvent aussi disposer de données secondaires propices à de nouvelles analyses. Vous pouvez aussi demander à ces organismes de procéder à une analyse spéciale de leurs données existantes.

La collecte de données primaires utiles à la conception d'un projet peut être plus coûteuse et exiger une plus grande expertise de la recherche en termes de conception d'outil, d'organisation des entretiens et de saisie des données. Elle permet cependant de spécifier l'information voulue et les filles précises auprès desquelles elle doit être collectée, y compris leur localité. L'Outil 1.1 proposé dans cet ouvrage consiste en un simple instrument quantitatif de collecte d'information de base auprès des filles, utile à l'élaboration d'un programme. À noter : lors de la conduite d'entretiens, il convient de prêter rigoureusement attention aux directives éthiques de conduite de la recherche, en ce qui concerne notamment le consentement et la confidentialité (voir les Ressources).

\section{Analyse}

L'analyse des fréquences ou des tableaux croisés sur les variables d'intérêt du Tableau 1 peut mettre en lumière les groupes ou les circonstances des filles qui méritent une attention programmatique. Par exemple, une population dans laquelle un pourcentage significatif de filles n'aurait jamais fréquenté l'école ou n'aurait atteint que de faibles niveaux de scolarité pourrait justifier une attention et une considération programmatique particulière. Les Figures 1, 2 et 3 reposent sur les données récentes de sept régions d'Éthiopie ${ }^{6}$ obtenues sur la base de questions similaires à celles de l'Outil 1.1. Ces figures indiquent le pourcentage de jeunes qui n'ont jamais fréquenté l'école et, pour ceux scolarisés, le nombre moyen d'années de scolarité. La Figure 1 révèle qu'environ un quart des adolescents des milieux ruraux n'ont jamais fréquenté l'école. La Figure 2 indique un niveau de scolarité global particulièrement faible, inférieur à quatre années en moyenne, parmi les jeunes des milieux ruraux. La Figure 3 présente le pourcentage de jeunes qui n'ont jamais fréquenté l'école, en les répartissant toutefois suivant qu'ils ont déjà été mariés ou non. Cette ventilation révèle d'importantes différences de participation au processus scolaire entre les adolescents qui n'ont jamais été mariés et ceux qui l'ont été. De telles analyses peuvent en dire long sur les filles, sur les endroits où les trouver et sur leurs besoins.

6 Erulkar A, Ferede A, Ambelu W, et al. 2010. “Ethiopia Young Adult Survey: A Study in Seven Regions," Addis Ababa: Population Council et UNFPA, septembre. Les données de cette étude ont été collectées dans les zones urbaines et rurales de sept régions: Addis Abeba, Afar, Amhara, Beneshangul-Gumaz, Oromia, Région des nations, nationalités et peuples du Sud (SNNPR) et Tigré. 
FIGURE 1 Pourcentage des adolescents éthiopiens de 12 à 19 ans n'ayant jamais fréquenté l'école, par sexe et milieu de résidence urbain ou rural $(n=6398)$

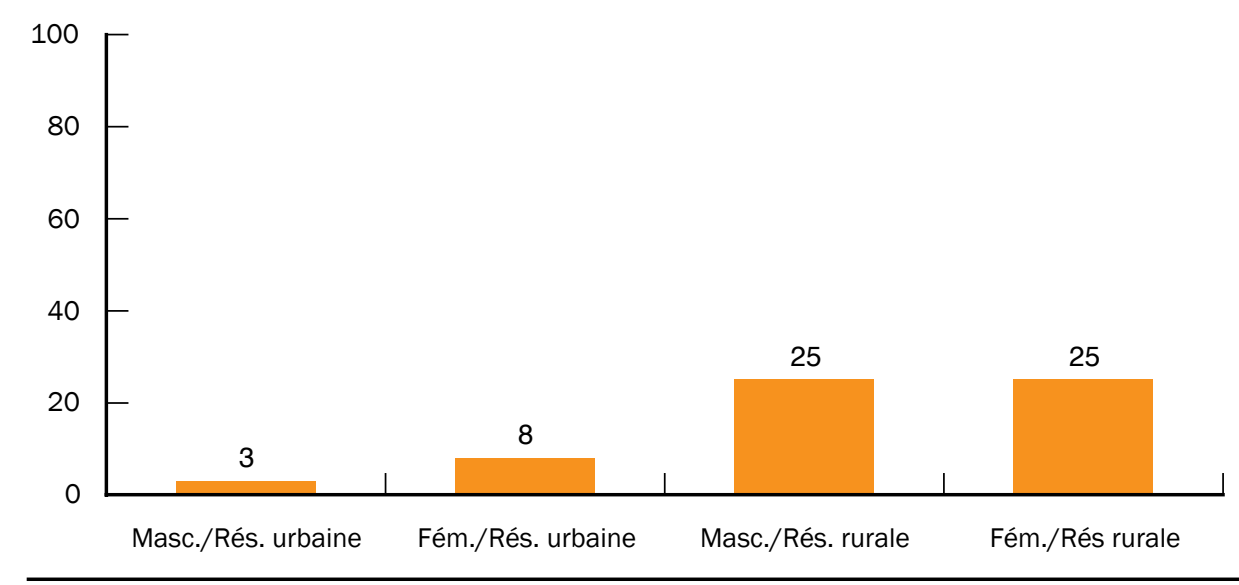

Source : Ethiopia Young Adult Survey (2009) ; données pondérées.

FIGURE 2 Nombre moyen d'années de scolarité des adolescents éthiopiens de 15 à 19 ans, par sexe et milieu de résidence urbain ou rural $(n=3662)$

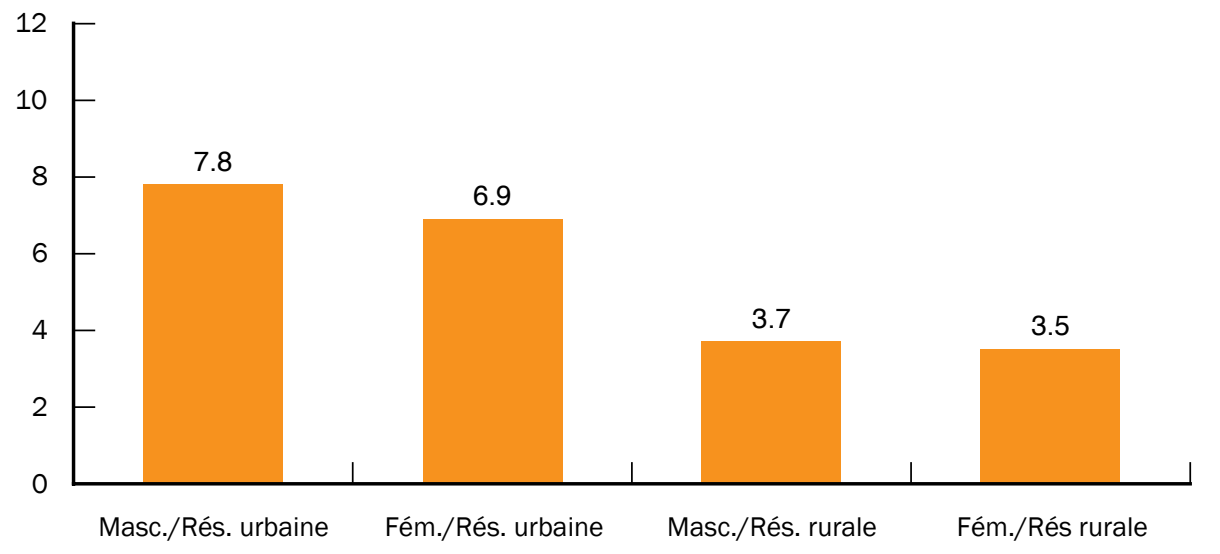

Source : Ethiopia Young Adult Survey (2009) ; données pondérées.

FIGURE 3 Pourcentage des adolescents éthiopiens de 12 à 19 ans n'ayant jamais fréquenté l'école, par sexe, milieu de résidence urbain ou rural et état matrimonial $(n=6398)$

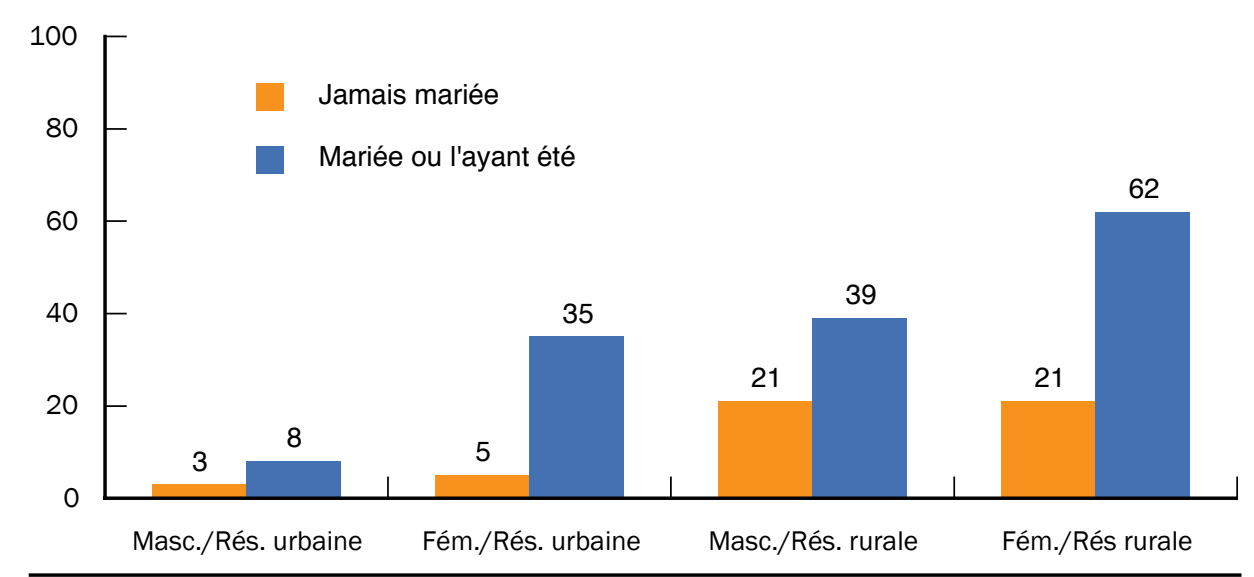

Source : Ethiopia Young Adult Survey (2009) ; données pondérées. 


\section{Identification des catégories de filles dans les milieux ruraux: ce que nous disent les données}

Certaines organisations mettent spécifiquement l'accent sur les filles les plus défavorisées, tandis que d'autres se concentrent sur la population générale des jeunes. Celles vouées aux initiatives de leadership sont parfois plus susceptibles de viser les populations d'élite ou relativement plus avantagées, comme les étudiants universitaires. Quoi qu'il en soit, il est important de définir les priorités de l'organisation et d'identifier les groupes cibles en conséquence. Les données peuvent servir à définir et différencier ces groupes.

Les organisations qui s'intéressent à la jeunesse rurale " générale " peuvent choisir de travailler avec la population scolarisée, qui représente la majorité. D'après les données, trois quarts des jeunes des milieux ruraux auraient fréquenté l'école. Si l'on voulait toutefois atteindre la jeunesse rurale générale " type ", les données laissent par ailleurs entendre qu'il vaut mieux concevoir les programmes au niveau primaire et non secondaire, car la majorité des jeunes n'achèvent que quelques années de l'enseignement primaire et n'accèdent jamais au niveau secondaire.

Qu'un jeune ait ou non l'âge correspondant à celui de sa classe représente aussi un facteur important à considérer. Si la majorité des jeunes des milieux ruraux vont à l'école primaire, beaucoup sont en âge de fréquenter l'enseignement secondaire. Une classe peut couvrir plusieurs âges, laissant entendre que le contenu d'un programme proposé au niveau primaire doit être adapté à l'âge des élèves et que l'hétérogénéité de la classe doit être prise en compte.

Les programmes axés sur les jeunes les plus défavorisés peuvent choisir de cibler ceux qui n'ont jamais fréquenté l'école. Un bon moyen de cerner les filles de cette catégorie serait de cibler les adolescentes mariées, parmi lesquelles une proportion estimée à deux tiers n'a jamais été scolarisée. Le Tableau 2 illustre et suggère un mode de différenciation et d'opérationnalisation de différents groupes cibles de filles des milieux ruraux selon trois indicateurs : situation scolaire, niveau de scolarité atteint et mariage. Il y aura, certes, toujours des cas exceptionnels, mais nous suggérons ces trois indicateurs comme utiles à la différenciation des niveaux de vulnérabilité parmi les jeunes Éthiopiennes des milieux ruraux.

TABLEAU 2 Catégorisation des filles sur la base d'indicateurs de scolarisation et de mariage (illustration)

\begin{tabular}{llll}
\hline CATÉGORIE DE JEUNESSE/ & \multicolumn{2}{c}{ Indicateur de scolarisation } & \\
\cline { 2 - 3 } FILLE RURALE & Situation scolaire & Niveau atteint & Indicateur de mariage \\
\hline Jeunesse relativement " favorisée " & Scolarisée & Enseignement secondaire & Jamais mariée \\
Jeunesse " générale " ou moyenne ${ }^{1}$ & Scolarisée & Niveau primaire & Jamais mariée \\
Jeunesse vulnérable & Jamais scolarisée & Aucune scolarisation & Jamais mariée \\
Jeunesse la plus vulnérable & ou déscolarisée & ou quelques années & Mariée \\
\hline
\end{tabular}

${ }^{1}$ La jeunesse " moyenne " désigne celle formant la majorité des jeunes. 
Dès le moment où un groupe cible a été défini, vous pouvez " dessiner le portrait " des filles considérées à l'aide des données, qui constituent votre base factuelle. Les données d'enquête comme celles des études qualitatives sont utiles à l'élaboration de ce portrait du groupe de filles à atteindre. La collecte et l'analyse de données qualitatives selon les méthodes de recherche traditionnelles peuvent prendre du temps et poser un défi aux gestionnaires des programmes. Nous suggérons l'élaboration de simples outils pour mettre en lumière la problématique fondamentale de la vie des filles et aider ainsi à les comprendre ainsi que leurs besoins programmatiques. Nous recommandons en outre le recours aux entretiens individuels en profondeur rapides plutôt qu'aux groupes de discussion. Les entretiens en profondeur tendent à éclaircir davantage l'information et les circonstances personnelles. Dans les groupes de discussion, les jeunes hésitent souvent à s'exprimer devant les autres et tendent parfois à donner des réponses normatives, socialement acceptables.

Formez vos intervieweuses à poser des questions ouvertes, puis à approfondir les réponses reçues en demandant par exemple : "Que peuxtu me dire d'autre à propos de cela ? " ou bien "Pourrais-tu me donner un exemple de situation où cela t'est arrivé ? " Encouragez le récit de la répondante : vous y trouverez souvent les données les plus utiles à l'élaboration des programmes. Si possible, enregistrez l'entretien sur cassette et transcrivez-le, en ce qui concerne les parties critiques au moins, verbatim.

L'Outil 1.2 offre un exemple de questionnaire d'entretien en profondeur rapide avec les filles. Cet exemple particulier se concentre sur l'expérience de la scolarisation. Le format peut cependant être adapté ou élargi pour inclure différents thèmes, comme les relations, le mariage, l'expérience sexuelle et le travail rémunéré ou non.

\section{ÉTUDE DE CAS : Identification des adolescentes mariées au centre des programmes}

En 2002, le Population Council a commencé à développer des programmes à l'intention des adolescents d'Éthiopie. Les gestionnaires savaient dès le départ qu'ils voulaient cibler les jeunes les plus défavorisés. Ils ont par conséquent sélectionné les segments pauvres ou défavorisés de milieux urbains aussi bien que ruraux pour mener leur recherche formative sur les adolescents. En 2003, des enquêtes ont été entreprises parmi les adolescentes et adolescents des bas quartiers d'Addis Abeba et des zones rurales pauvres de la région d'Amhara, seconde grande région d'Éthiopie.

Les gestionnaires du Population Council et du ministère éthiopien de la Jeunesse et des Sports ont utilisé ces enquêtes pour déterminer les groupes de jeunes les plus vulnérables de ces milieux. Ils ont présumé que si un jeune adolescent de 10 à 14 ans ne fréquentait pas l'école, il s'agissait probablement d'un jeune en position particulièrement vulnérable. Dans les deux contextes, ruraux et urbains, les filles se sont avérées plus susceptibles d'être déscolarisées que les garçons. L'examen des jeunes les plus susceptibles de ne pas fréquenter l'école a révélé deux groupes. Dans les milieux ruraux, il s'agissait des adolescentes mariées et dans les milieux urbains, des migrantes de la campagne à la ville, employées pour beaucoup aux travaux domestiques. L'analyse approfondie a dévoilé l'extrême isolement social des deux groupes, beaucoup ayant déclaré ne pas avoir d'amies. Par conséquent, le Population Council et le ministère de la Jeunesse et des Sports ont entrepris la mise au point de programmes destinés aux adolescentes mariées et aux domestiques enfants/migrantes de la campagne à la ville. 


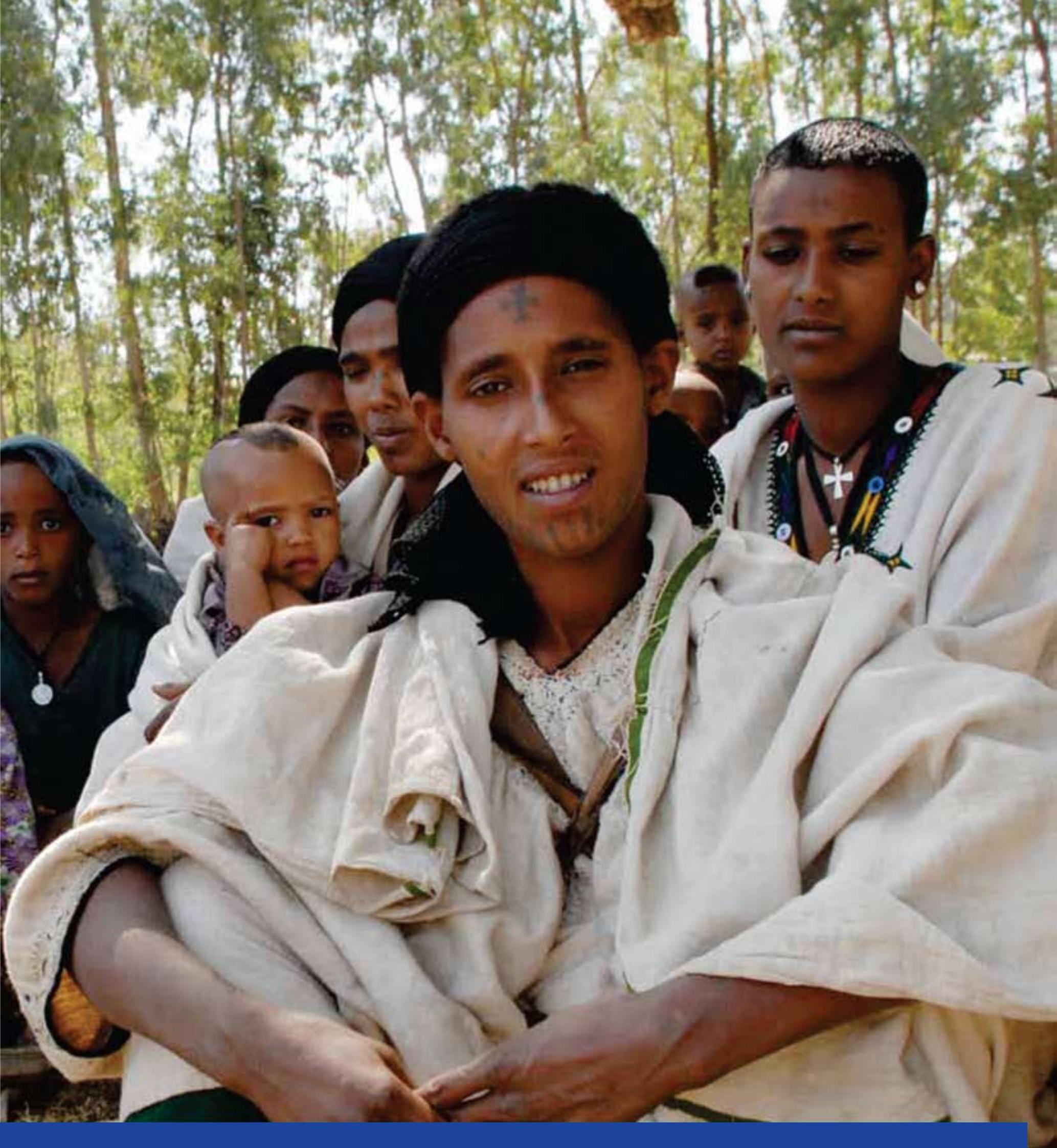





\section{CHAPITRE 2 \\ CONCEPTION PROGRAMMATIQUE : FAÇONNER L'APPROCHE}

$\mathrm{T}$

rois grands composants entrent en jeu dans la conception programmatique : le lieu, la stratégie (ou mode de prestation) et le contenu. Le lieu désigne l'endroit où se déroule le programme. La stratégie (ou mode de prestation) concerne la modalité ou les activités qui servent de véhicule au contenu programmatique. Enfin, le contenu représente les messages spécifiques, l'information ou le renforcement de compétences visés par le programme. Les données sont utiles à la définition des trois.

\section{Lieu du programme}

Il existe deux grandes stratégies d'identification du lieu de déploiement d'un programme pour filles. L'une consiste à établir le programme à l'endroit où les filles se trouvent déjà. II s'agit là de l'une des stratégies les plus répandues, en général, dans la programmation pour les jeunes et qui a contribué au grand nombre de programmes de jeunes implantés dans les écoles. Certaines catégories de jeunes ne sont cependant pas rattachées aux institutions ou ne fréquentent pas régulièrement les mêmes endroits. En particulier, certaines catégories de filles - celles mariées et les domestiques, notamment - se retrouvent rarement en dehors de leur propre foyer ou de leur lieu d'emploi. Les programmes de jeunes doivent alors créer un nouveau lieu de rencontre pour les filles. La déscolarisation de nombreux jeunes et la difficulté de les joindre ont mené, dans le passé, à d'importants investissements dans les centres de jeunesse. On y verra un exemple de création de lieu de déploiement d'un programme. Mais il existe probablement d'autres moyens d'adaptation localement plus appropriés et responsables que la construction d'une toute nouvelle infrastructure.

Les données peuvent aider à déterminer les endroits où les filles se trouvent peut-être déjà et s'il convient ou non de créer de nouveaux emplacements pour le programme. L'Outil 1.1 comporte une question invitant la fille à indiquer si elle s'est rendue dans un lieu de culte, un centre de jeunesse ou un établissement de santé durant les 12 derniers mois. Les réponses à cette question apportent une information utile à l'identification des lieux d'implantation envisageables pour les programmes destinés aux filles.

La Figure 4 présente les résultats d'une enquête récente concernant l'exposition institutionnelle des filles des milieux ruraux durant les 12 derniers mois. ${ }^{7}$ Les responsables programmatiques qui s'intéressent largement aux filles des milieux ruraux d'Éthiopie peuvent considérer l'établissement d'initiatives liées aux institutions religieuses : les trois quarts

7 Erulkar A, Ferede A, Ambelu W, et al. 2010. "Ethiopia Young Adult Survey: A Study in Seven Regions," Addis Abeba: Population Council et UNFPA, septembre. Les données de cette étude ont été collectées dans les zones urbaines et rurales de sept régions : Addis Abeba, Afar, Amhara, Beneshangul-Gumaz, Oromia, Région des nations, nationalités et peuples du Sud (SNNPR) et Tigré. 
FIGURE 4 Question d'enquête et résultats concernant l'exposition institutionnelle des filles de 12 à 19 ans des milieux ruraux d'Éthiopie

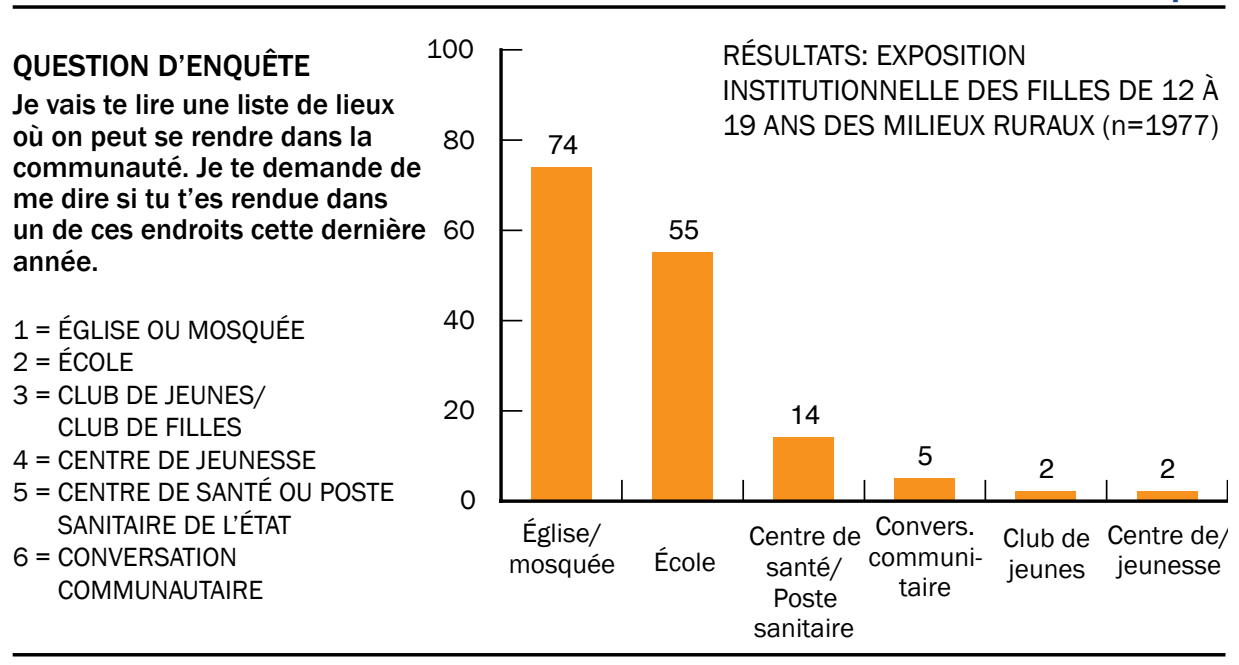

Source : Ethiopia Young Adult Survey (2009) ; données pondérées.

des filles visées ont en effet été exposées à de tels endroits durant les 12 derniers mois si pas durant la dernière semaine écoulée. Vient ensuite l'école, qui " capte " environ la moitié des filles des milieux ruraux. Les initiatives en centre de jeunesse n'atteindraient vraisemblablement que très peu de filles dans les milieux ruraux. L'enquête révèle en effet le très faible nombre de filles qui fréquentent ces centres, en raison, probablement, de la distance à parcourir.

La Figure 5 présente les mêmes résultats d'exposition institutionnelle des filles des milieux ruraux, répartis en fonction de leur état matrimonial. Les emplacements ou " points de capture " institutionnels existants sont différents suivant que les filles sont mariées ou non. Pour les deux groupes, les institutions religieuses demeurent de bons lieux d'accès. L'école est un lieu d'accès moins efficace aux filles mariées, contrairement aux établissements de santé et aux conversations communautaires où les perspectives sont légèrement meilleures pour ce groupe.

FIGURE 5 Résultats concernant l'exposition institutionnelle des filles de 12 à 19 ans des milieux ruraux d'Éthiopie, en fonction de l'état matrimonial

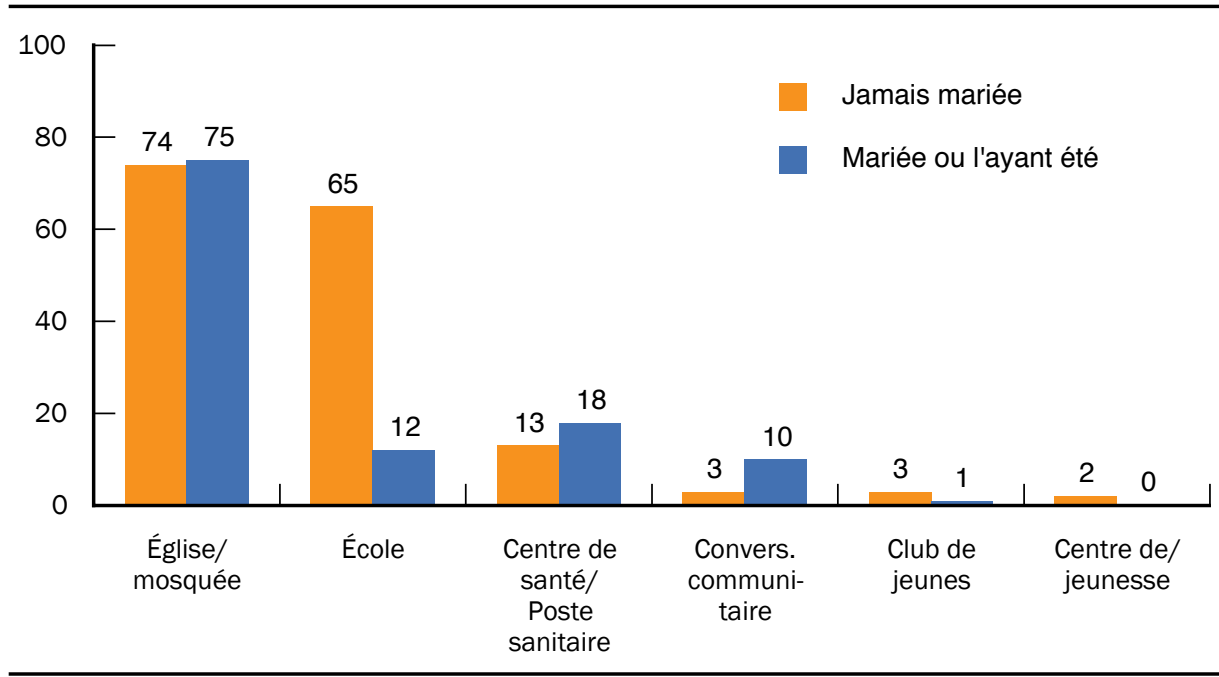

Source : Ethiopia Young Adult Survey (2009) ; données pondérées. 
De fait, mises à part les institutions religieuses et, peut-être, les écoles primaires, il n'existe guère de lieux ou " points d'accès " programmatique existants concernant les filles des milieux ruraux. En bien des endroits d'Éthiopie rurale, les lieux ou plates-formes programmatiques devront être créés.

\section{Stratégie / Mode de prestation}

Le Tableau 3 présente quelques exemples de modes de prestation ou stratégies programmatiques. Les stratégies médiatiques, à travers la radio ou les journaux, offrent des modes de prestation répandus lorsque la couverture de la radio ou de la télévision est vaste et que les populations alphabétisées ont accès à la presse écrite ou à d'autres formes d'information imprimée. Les approches en établissement conviennent généralement mieux en milieu urbain et exigent souvent l'établissement de nouvelles installations ou structures. Dans les milieux ruraux, où la distance représente un facteur avec lequel compter, les programmes en établissement sont moins réalistes (comme le révèle la Figure 5). Les approches communautaires sont probablement les mieux adaptées aux milieux ruraux d'Éthiopie, étant donné les distances considérables, l'infrastructure limitée et la faible couverture des véhicules médiatiques tels que la radio. Seuls $35 \%$ des foyers ruraux où vivent les filles possèdent en effet un poste de radio.

Les données appropriées peuvent aussi suggérer de bonnes stratégies d'apport de contenu. Les approches communautaires étant souvent les plus appropriées dans les contextes ruraux, les données relatives aux réseaux sociaux - amitiés, groupes sociaux ou affiliations - sont extrêmement utiles à l'orientation des programmes. La Figure 6 présente les données obtenues des filles des milieux ruraux sur leurs réseaux d'amitiés et leur co-résidence avec leurs parents. Le tableau correspondant illustre

TABLEAU 3 Exemples de stratégies programmatiques

\begin{tabular}{|c|c|c|}
\hline TYPE DE STRATÉGIE & STRATÉGIE & EXEMPLE DE PROGRAMME POUR FILLES \\
\hline \multirow[t]{3}{*}{ Médias } & \multirow[t]{2}{*}{ Radio } & $\begin{array}{l}\text { Émission de radio communautaire RATSON consacrée aux } \\
\text { questions relatives aux filles. }\end{array}$ \\
\hline & & $\begin{array}{l}\text { Programme "Abu Gida " du BBC World Service Trust, } \\
\text { consacré aux questions qui affectent les filles. }\end{array}$ \\
\hline & Presse & $\begin{array}{l}\text { Journal mensuel de Save Your Generation Ethiopia (SYGE) } \\
\text { publié à l'intention des jeunes hommes et femmes. }\end{array}$ \\
\hline Approche en établissement & Refuge & Refuge OPRIFS pour filles victimes de sévices. \\
\hline \multirow[t]{6}{*}{ Approche communautaire } & $\begin{array}{l}\text { Éducation par } \\
\text { les pairs }\end{array}$ & $\begin{array}{l}\text { Pairs éducatrices Kulich des filles employées dans la } \\
\text { floriculture. }\end{array}$ \\
\hline & $\begin{array}{l}\text { Mentoring individuel } \\
\text { ou de groupe }\end{array}$ & $\begin{array}{l}\text { Groupes de mentoring PACT pour filles scolarisées et } \\
\text { déscolarisées, mettant l'accent sur l'autonomisation, } \\
\text { l'éducation et les moyens de subsistance. }\end{array}$ \\
\hline & $\begin{array}{l}\text { Conversations } \\
\text { communautaires }\end{array}$ & $\begin{array}{l}\text { Conversations communautaires d'EGLDAM sur la } \\
\text { mutilisation génitale des filles/l'excision, le mariage } \\
\text { précoce, l'enlèvement, etc. }\end{array}$ \\
\hline & \multirow{2}{*}{$\begin{array}{l}\text { Groupes de jeunesse, } \\
\text { clubs ou groupes } \\
\text { d'entraide }\end{array}$} & $\begin{array}{l}\text { Groupes de filles du Secours catholique ayant acquis } \\
\text { des terres à cultiver. }\end{array}$ \\
\hline & & $\begin{array}{l}\text { Groupes de filles des associations de solidarité féminine } \\
\text { (WSA) pour l'épargne et l'éducation à la santé génésique. }\end{array}$ \\
\hline & $\begin{array}{l}\text { Approches parent- } \\
\text { enfant }\end{array}$ & $\begin{array}{l}\text { Les associations de solidarité féminine (WSA) organisent } \\
\text { des dialogues familiaux pour la discussion de sujets tels que } \\
\text { la santé génésique, le VIH/sida et les moyens de subsistance. }\end{array}$ \\
\hline
\end{tabular}


FIGURE 6 Recours aux données relatives aux réseaux sociaux pour la suggestion de stratégies programmatiques à l'intention des filles de 12 à 19 ans des milieux ruraux d'Éthiopie

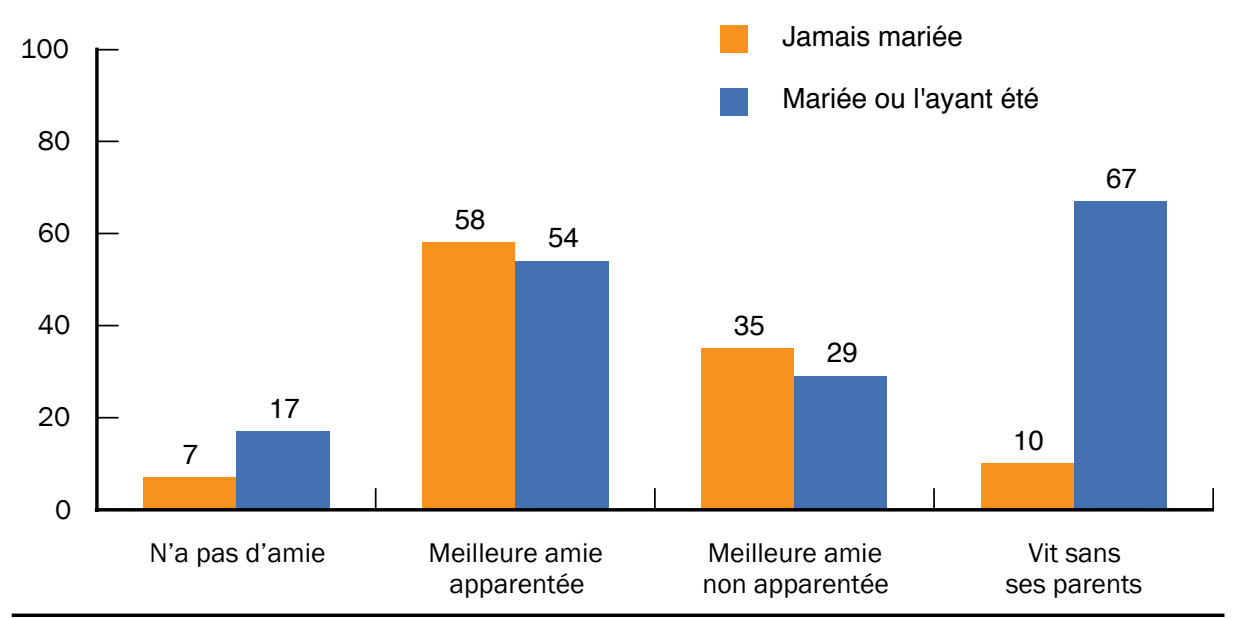

Source : Ethiopia Young Adult Survey (2009); données pondérées.

l'application des résultats de la recherche dans la détermination de l'approche communautaire la plus bénéfique possible.

L'éducation par les pairs a recours aux amies, les "pairs ", pour apporter un message aux filles ou renforcer leurs compétences. Les données ci-dessus indiquent que beaucoup de filles, surtout mariées, n'ont pas d'amies. Celles qui en ont forment en majorité leurs amitiés au sein même de la famille plutôt qu'avec des pairs non apparentées, comme on le supposerait souvent. Ces constatations laissent entendre que l'efficacité, ou la portée, de l'éducation par les pairs pourrait être limitée dans ces contextes. Le modèle de l'éducation par les pairs part du principe de la présence de pairs hors famille ; il est le plus efficace lorsque les jeunes disposent de réseaux sociaux vastes et divers et bénéficient d'un contact intense et varié avec leurs pairs. Les réseaux des filles des milieux ruraux sont relativement limités et fermés, laissant entendre une efficacité vraisemblablement restreinte du modèle de pair à pair. La leçon pourrait aussi être que les programmes devraient chercher à étendre et diversifier les réseaux de pairs des filles dans les milieux ruraux.

Si pas les pairs, qui pourrait offrir une stratégie utile à l'apport des programmes aux jeunes filles ? Une fille non mariée sur 10 en Éthiopie rurale vit sans ses parents, de même que la majorité des filles mariées. Indépendamment de leur état matrimonial, les adolescentes doivent pouvoir compter sur une personne adulte qui les aime. Cela d'autant plus que dans les contextes traditionnels, les jeunes filles occupent souvent un rang relativement faible au sein de la communauté et ont besoin d'une représentante, prête à les défendre, de plus haut rang. Aussi la stratégie programmatique peut-elle inclure une femme adulte appelée à assumer ce rôle et à agir au titre de défenseur potentielle des filles, que le contact soit établi ou non dans un contexte individuel ou de groupe.

Nous avons tiré parti des conclusions de la recherche sur les réseaux sociaux des filles des milieux ruraux pour développer une stratégie programmatique. Les résultats et les implications de nos constats évoquent une stratégie de mentoring sous la conduite d'une femme adulte, qui donne aux filles une connexion à une " amie " de plus haut rang, en combi- 
naison avec les groupes de filles, avec pour effet l'élargissement de leurs réseaux de pairs. Les données de communautés spécifiques de filles des milieux ruraux peuvent informer les gestionnaires des programmes de l'opportunité éventuelle d'autres stratégies. Par exemple, dans les communautés où le mariage précoce est rare et où la plupart des filles vivent encore avec leurs parents, les modèles parent-enfant peuvent être justifiés et efficaces. Là où les filles sont largement scolarisées et ont accès à de plus vastes réseaux de pairs, les groupes de filles peuvent être suggérés. Étant donné le faible statut des filles dans de nombreuses communautés rurales toutefois, les approches autonomes de pair à pair risquent de manquer de prise et d'impact sur les normes sociales en l'absence d'une forme d'affiliation à des adultes de plus haut rang.

\begin{tabular}{|c|c|c|c|}
\hline $\begin{array}{l}\text { Constatation de } \\
\text { la recherche }\end{array}$ & $\begin{array}{l}\text { Caractéristique } \\
\text { reflétée ou suggérée }\end{array}$ & $\begin{array}{l}\text { Implication } \\
\text { programmatique }\end{array}$ & $\begin{array}{l}\text { Exemple de stratégie } \\
\text { programmatique }\end{array}$ \\
\hline $\begin{array}{l}\text { AMIES } \\
\text { 1. Dans les milieux ruraux, } \\
\text { environ une fille sur } 10 \text { n'a } \\
\text { pas d'amies; une sur cinq } \\
\text { parmi les filles mariées. } \\
\text { 2. Parmi les filles qui ont } \\
\text { des amies, la plupart sont } \\
\text { internes à la famille. }\end{array}$ & $\begin{array}{l}\text { Les réseaux sociaux } \\
\text { des filles des milieux } \\
\text { ruraux sont axés sur } \\
\text { les membres de la } \\
\text { famille. }\end{array}$ & $\begin{array}{l}\text { L'éducation par les pairs peut } \\
\text { être inefficace parmi les filles } \\
\text { des milieux ruraux qui n'ont } \\
\text { guère d'amies ou de pairs en } \\
\text { dehors du foyer. }\end{array}$ & $\begin{array}{l}\text { Renforcer les réseaux de } \\
\text { pairs extérieurs : fonder } \\
\text { des clubs de filles où elles } \\
\text { puissent rencontrer leurs } \\
\text { pairs en dehors de la } \\
\text { famille. }\end{array}$ \\
\hline $\begin{array}{l}\text { PARENTS } \\
\text { 3. La plupart des filles } \\
\text { mariées ne vivent pas } \\
\text { avec leurs parents. }\end{array}$ & $\begin{array}{l}\text { Les filles mariées n'ont } \\
\text { peut-être pas d'adulte } \\
\text { aimante dans leur vie. }\end{array}$ & $\begin{array}{l}\text { Les approches parent-enfant } \\
\text { n'atteignent pas les filles } \\
\text { mariées et, quel que soit leur } \\
\text { état matrimonial, les filles } \\
\text { doivent pouvoir compter sur une } \\
\text { adulte aimante. }\end{array}$ & $\begin{array}{l}\text { Établir une relation avec } \\
\text { une adulte aimante qui } \\
\text { puisse les représenter } \\
\text { et les défendre : recourir } \\
\text { aux mentors plutôt qu'aux } \\
\text { pairs. }\end{array}$ \\
\hline
\end{tabular}

\section{ÉTUDE DE CAS : Recourir à la stature des mentors pour vaincre les résistances}

Meserete Hiwot (" la base de la vie " en amharique) est un programme pour adolescentes mariées ou l'ayant été dans la région rurale d'Amhara, mis en œuvre par le Bureau régional d'Amhara pour les femmes, les enfants et les jeunes (ancien Bureau régional d'Amhara de la jeunesse et des sports). Ce programme réunit des groupes d'adolescentes mariées ou divorcées, sous la conduite d'une femme mentor adulte. Cette femme est recrutée au sein de la communauté locale. II s'agit fréquemment d'une leader locale bien connue de l'endroit.

Les adolescentes mariées sont souvent soumises à la stricte autorité de leur mari ou de leur belle-famille, dont certains membres limitent parfois leurs relations avec des amies ou leur affiliation à d'autres groupes sociaux. Les gestionnaires de Meserete Hiwot ont cependant pensé que le recours à des mentors de plus haut rang, par opposition aux pairs éducatrices, offrirait une stratégie efficace apte à convaincre les familles résistantes d'autoriser les filles à participer aux rencontres. Les études de suivi de Meserete Hiwot reflètent la valeur ajoutée de ces mentors dans le renversement des obstacles à la participation :

" [D'abord] mon mari ne m'avait pas autorisée à assister à la réunion. Notre mentor est venue le voir et l'a convaincu en privé... " (Fille mariée, Gondar Nord, 21 ans, non scolarisée, un enfant)

"Ma mère n'était pas contente. Elle voulait que je vende de l'alcool plutôt que d'assister aux rencontres. J'ai fait venir notre mentor pour la convaincre et, au début, je lui racontais aussi ce dont nous avions parlé à la réunion. "(Fille divorcée, Gojam Ouest, 18 ans, 3 années de scolarité, pas d'enfants) 


\section{SIMPLES QUESTIONS POUR FAIRE PARLER LES FILLES}

- Parle-moi de toi.

- À quels moments de ta vie es-tu la plus heureuse ? Qu'est-ce qui te rend heureuse ?

- Quand te sens-tu soutenue ? Qui te donne son soutien et quel genre de soutien ou de conseil reçoistu de cette ou de ces personnes ? Peux-tu me donner un exemple?

- Parle-moi d'un moment où tu as eu besoin de soutien ou que tu as désiré en avoir. Que s'est-il passé ?

- Parle-moi d'un moment où tu aurais voulu t'exprimer, dire quelque chose, mais tu as eu trop peur de le faire. Que s'est-il passé ? De quoi avais-tu trop peur de parler ? Pourquoi ?

- Quand te sens-tu le plus en sécurité ? Peux-tu me donner un exemple d'une circonstance où tu te sens en sécurité ?

- Parle-moi d'un moment où tu t'es sentie en danger ou effrayée. Que s'est-il passé ?

- Y a-t-il jamais eu un moment où tu as été forcée de faire quelque chose que tu ne voulais pas, contre ta volonté ? Donne-moi un exemple. Que s'est-il passé ?

\section{Contenu}

Le contenu des programmes doit être déterminé sur la base 1) des demandes des filles, 2) de la voix des filles et 3) du jugement professionnel de spécialistes. Les filles visées peuvent être interrogées directement sur l'information ou les services qu'elles aimeraient obtenir du programme. Les filles ellesmêmes sont sources d'excellentes idées à considérer dans les programmes. Cela dit, toutes leurs suggestions ne sont pas toujours réalistes, compte tenu du financement ou de la mission du projet, ou même souhaitables.

Les demandes directes des filles diffèrent de leur " voix ". La " voix " des filles est leur histoire : ce qu'elles disent de leur vie et de leur vécu. Inviter les filles à raconter leur vie et leur histoire peut être fort utile au façonnement du contenu des programmes qui leur sont destinés. Par exemple, si les filles ne demandent pas directement d'aide contre la violence de leurs circonstances, leur histoire peut être révélatrice. Pour utiliser la voix des filles, il faut recueillir leur histoire par le biais de techniques qualitatives. Cela fait, les professionnels ou gestionnaires (en collaboration avec les filles peut-être) peuvent extraire des récits obtenus les besoins des filles auxquels un programme pourrait répondre. L'histoire des filles peut être recueillie à l'aide de simples questionnaires tels que l'Outil 1.2 décrit au chapitre 1. Cet outil peut être modifié et adapté suivant le besoin (voir l'encadré "Simples questions pour faire parler les filles ").

L'histoire des filles peut révéler de précieux indices quant à leurs besoins de soutien, leurs besoins d'information ou les lacunes de leurs compétences, autant de thèmes que les programmes peuvent chercher à résoudre. Le schéma ci-dessous illustre comment le récit de la vie et du vécu des filles peut donner aux responsables programmatiques d'importants indices ou signes quant au contenu à couvrir.

Enfin, l'opinion professionnelle et la connaissance experte sont des guides essentielles aussi. La raison en est que les jeunes ne savent pas toujours ce qui leur convient le mieux d'un point de vue affectif ou de développement. Ainsi, les filles qui n'ont pas été socialisées pour s'adresser aux hommes adultes ne savent peut-être pas qu'elles doivent apprendre les compétences qui leur permettent de le faire ; celles sans extrait d'acte de naissance ne sont peut-être même pas conscientes de leur existence. Nous exposons plus bas quelques domaines de contenu parfois omis par les filles et que les programmes de jeunes plus courants, consacrés aux compétences de vie, à la santé génésique et au VIH/sida, tendent à ne pas 
couvrir. Remarquez que nous ne mettons pas l'accent sur les domaines programmatiques que nous savons déjà couverts par les programmes de jeunesse existants d'Éthiopie.

\section{VOIX/HISTOIRE DE LA FILLE}

" J'ai peur qu'on sache que j'ai mes règles. J'ai tellement peur de tacher mes vêtements. Je ne peux pas courir. Si j'avais une serviette, je pourrais faire tout ce que je veux... Quand j'allais à l'école, si j'avais un examen, j'en parlais à la maîtresse pour pouvoir le présenter à un autre moment, ou bien je risquais d'avoir de plus mauvais points que les autres élèves. Je ne pouvais pas faire de gymnastique. Je marchais lentement. " Fille d'Amhara, 17 ans, divorcée

\section{AJOUT AU PROGRAMME}

- Information sur la menstruation et sa gestion.

- Apport de matériel de gestion de la menstruation, comme les serviettes réutilisables localement produites, fixées à l'aide de velcro.

\section{VOIX/HISTOIRE DE LA FILLE}

"Quand je rentrais chez moi le soir, un homme de mon village m'a demandé de l'accompagner. J'avais trop peur pour refuser. Arrivés près de mon village, il a ouvert sa braguette... J'ai couru vers un endroit plus éclairé et je lui ai demandé en criant ce qu'il essayait de faire. "Fille d'Amhara, 18 ans, non mariée

\section{AJOUT AU PROGRAMME}

- Compétences nécessaires aux filles des milieux ruraux pour communiquer avec les personnes et les hommes plus âgés. - Sensibilisation au harcèlement, au viol et à la contrainte.

\section{CONTENU COURAMMENT OMIS DES PROGRAMMES POUR FILLES}

\section{Citoyenneté et sécurité des filles}

- Sensibilisation et assistance à l'obtention d'extraits d'acte de naissance ou d'autres formes d'identification.

- Amélioration de la sécurité des filles et de leur accès aux espaces sûrs.

- Sensibilisation aux ressources disponibles dans la communauté ou dans le voisinage des filles (cliniques, refuges, assistance juridique, police, etc.)

\section{Soutien scolaire}

- Assistance à l'acquisition ou apport de matériel et uniformes scolaires.

- Enseignement individuel.

- Temps protégé réservé aux devoirs plutôt qu'aux travaux domestiques.

Moyens de subsistance et autonomisation économique

- Éducation financière, y compris à l'épargne.

- Accès aux lieux sûrs où placer ses économies.

- Promotion et facilitation de la propriété terrienne.

Santé, y compris génésique

- Hygiène et nutrition.

- Visites médicales de base, exposition des filles au système de santé.

- Menstruation et gestion de la menstruation.

- Viol et autres formes de contrainte sexuelle.

- Pouvoir au sein des relations sexuelles et violence du partenaire intime.

- Soutien des victimes de la violence.

- Expérience de la grossesse et de la maternité.

\section{Créativité et expression personnelle}

- Loisirs et sports.

- Arts visuels et danse. 


\section{Principes de la programmation}

Lors de la phase conceptuelle de lieu, de stratégie et de contenu des programmes destinés aux filles des milieux ruraux, nous recommandons l'adhésion à quelques principes de bonne programmation. La liste ci-dessous énonce quelques-uns des principes qui nous paraissent clés dans la conception programmatique, dans le contexte des pays en développement surtout :

- Tirer au maximum parti de l'infrastructure existante.

- Atteindre une grande proportion des filles présentes dans votre zone géographique.

- Concevoir dans une perspective de durabilité.

- Durable ne veut pas dire gratuit. Éviter le recours aux bénévoles et rémunérer le personnel conformément aux catégories d'emploi similaires dans le contexte local.

- Les programmes doivent être simples. Éviter la complexité.

\section{Tirer au maximum parti de l'infrastructure existante.}

Dans la mesure du possible, nous préconisons l'usage de l'infrastructure disponible au niveau de la communauté, y compris en ce qui concerne les ressources humaines, les établissements et les terres. ${ }^{8}$ Que les programmes de mentoring recrutent par exemple les leaders féminines locales, qui connaissent la communauté et ont le plus de chances de réussir. De même, on utilisera les structures existantes comme espaces de rencontre, plutôt que d'investir dans de nouvelles installations qui ne seront accessibles qu'aux filles du voisinage immédiat. Les zones rurales présentent l'avantage de vastes espaces ; les rencontres sous un arbre peuvent, et doivent, être la règle. Parmi les autres ressources communautaires existantes potentielles, on considérera les associations et les ligues de jeunes, de même que les institutions religieuses, qui bénéficient d'une vaste portée dans les milieux ruraux.

Atteindre une grande proportion des filles présentes dans votre zone géographique.

Selon le modèle programmatique suivi, il convient généralement d'atteindre une grande proportion des filles admises au programme, au sein de la zone même du projet. La raison en est double. En faisant profiter une grande proportion de filles des améliorations apportées à travers les programmes, on a plus de chances de produire un impact positif significatif au niveau de la population plutôt qu'à celui de quelques filles sélectionnées seulement. Cette stratégie peut ensuite changer les normes sociales pour une génération de filles dans la zone du projet. En ne soutenant par contre que quelques filles sélectionnées, ces filles deviennent souvent " marginales " au sein de leur communauté, qu'elles finissent alors par quitter. Par exemple, les programmes de bourses dont ne bénéficient que

8 Hughes J, McCauley A. 1998. “Improving the fit: Adolescents' Needs and Future Programs for Sexual and Reproductive Health in Developing Countries" Studies in Family Planning, 29(2): 233-245. 
quelques élues aboutissent souvent sur le départ des bénéficiaires, en quête de meilleures opportunités, avec peu d'effet restant au sein de la communauté d'origine. En revanche, le soutien d'une communauté entière de filles, pour qu'elles bénéficient par exemple de juste deux années de plus de scolarité, produit un impact à long terme sur la santé et le bienêtre des enfants et des familles de la communauté, et ce pendant de nombreuses années.

\section{Concevoir dans une perspective de durabilité.}

Dès le début du processus de conception, les gestionnaires doivent prêter attention aux facteurs d'échelle et de durabilité financière. II convient dès lors de déterminer les coûts des interventions dès la phase conceptuelle et le coût par bénéficiaire doit être estimé. Certaines stratégies propices à la durabilité d'un modèle incluent le recours aux échelles salariales locales, semblables à celles de l'état. Nous voyons dans les échelles salariales de l'état le reflet de la réalité économique du pays. Leur utilisation maximise les chances de durabilité d'un programme. L'usage maximum de l'infrastructure existante, tel que décrit plus haut, offre une autre stratégie de durabilité.

\section{Durable ne veut pas dire gratuit. Éviter le recours aux bénévoles.}

Nous estimons utile de payer un salaire de subsistance en compensation du travail accompli, surtout dans les milieux pauvres. II a été démontré que le recours aux volontaires s'avère extrêmement coûteux pour les programmes, en termes de coûts de formation surtout. ${ }^{9}$ La qualité et la continuité des programmes souffrent lorsque le personnel change souvent et l'absence de rémunération donne à penser que le travail n'est ni valorisé, ni utile. Nous sommes d'avis que le soutien des filles des milieux ruraux est un travail précieux qu'il convient, en tant que tel, de rémunérer, selon des échelles établies de manière pratique et réaliste toutefois. Cela sans compter que le personnel rémunéré constitue une démonstration explicite de moyens de subsistance positifs ouverts aux filles et aux femmes.

\section{Les programmes doivent être simples. Éviter la complexité.}

Bien des gestionnaires sont attirés par les programmes " globaux " ou " holistiques " qui couvrent tout un éventail de problèmes, car ils semblent répondre aux multiples défis que doivent relever les jeunes. II n'est donc pas rare de voir une organisation mettre en œuvre des programmes qui embrassent plusieurs secteurs, comme la santé génésique, l'éducation et les moyens de subsistance. Or les projets complexes à composants multiples sont difficiles à gérer et souvent de faible qualité. II est de plus extrêmement difficile de les élever au niveau suivant.

La simplicité des modèles maximise les chances de qualité et d'impact. Si d'autres composants sont requis en dehors de l'expertise de l'organisation, il vaut mieux opérer en partenariat avec d'autres organismes mieux armés pour assurer les services nécessaires. Ainsi, de nombreuses initiatives de subsistance ont échoué dans le cadre des organisations de santé

9 Janowitz B, et al. 2003. "The costs of programmes at selected youth centers in South Africa," FRONTIERS Report. Washington DC: Population Council.
La simplicité des modèles maximise les chances de qualité et d'impact. 
génésique. Le partenariat avec une organisation expérimentée et spécialisée dans ce domaine est recommandé, plutôt que de tenter une mise en œuvre qui dépasse l'expertise du programme. Dans l'ensemble, les conceptions programmatiques simples sont généralement plus rentables, plus efficaces et de meilleure qualité que celles extrêmement complexes. 


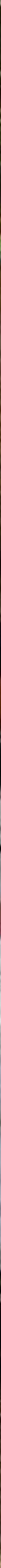

CHAPITRE 3

MISE EN CEUVRE : RESTER À L'ÉCOUTE DE LA SITUATION SUR LE TERRAIN ET PRENDRE DU RECUL POUR JUGER L'ENSEMBLE 



\section{CHAPITRE 3}

\section{MISE EN CEUVRE : RESTER À L'ÉCOUTE DE LA SITUATION SUR LE TERRAIN ET PRENDRE DU RECUL POUR JUGER L'ENSEMBLE}

a phase de conception terminée, le programme peut être mis en $œ u v r e$. Nous proposons et décrivons dans ce chapitre de simples méthodes et outils de suivi de cette mise en œuvre. Le suivi de programme aide les gestionnaires à 1) évaluer la fidélité au modèle, pour s'assurer que le programme est bien exécuté comme planifié, 2) suivre le progrès et le rythme de la mise en œuvre en la comparant au plan et 3) apporter les ajustements intermédiaires suivant différents facteurs de perception et feedback des filles, nouvelles perspectives découvertes au sujet des filles ou révélées par elles, difficultés rencontrées ou occasions présentées et tendances de sous- ou de sur-utilisation.

Les gestionnaires doivent reconnaître qu'ils ne sont pas omniscients. Il convient d'accepter dès le départ qu'un programme évoluera au fil du temps, à mesure de la découverte des filles des milieux ruraux, de leurs circonstances et de leur interaction avec le programme. On restera ouvert à l'idée d'un modèle évolutif et aux adaptations qui se justifieront au fil de l'expérience acquise. Pour maximiser la découverte et assurer une évolution efficace, les programmes doivent être suivis depuis plus d'un point de vue, à l'aide de données diverses, plutôt que dans une seule perspective ou à l'aide d'un outil unique.

Nous recommandons une combinaison de stratégies de suivi qui recueillent l'information à la base tout en examinant aussi les résultats au niveau global, selon des méthodes qualitatives aussi bien que quantitatives. Autrement dit, notre suggestion est celle d'une stratégie de suivi qui reste simultanément à l'écoute sur le terrain tout en prenant du recul pour juger l'ensemble.

\section{Rester à l'écoute sur le terrain}

La gestion programmatique efficace inclut un suivi fréquent des programmes sur le terrain, au niveau de la communauté. Personne ne peut véritablement comprendre le fonctionnement effectif d'un programme sur la base de rapports de seconde main. Les visites directes sur le terrain sont cruciales et leur importance ne doit pas être sous-estimée.

Si les visites et le dialogue avec le personnel et les bénéficiaires du programme sont inestimables, nous recommandons aussi, dans la mesure du possible, le recours à de simples outils de suivi qualitatif à l'occasion de ces rencontres. L'Outil 3.1 en est un exemple, utile au suivi auprès des bénéficiaires ou d'autres intéressés. Les données peuvent être collectées tous les trois à six mois, auprès d'un nombre limité de bénéficiaires (8 à 12) et autres intéressés, ainsi qu'auprès du personnel du programme. Elles permettent de tirer meilleur parti des visites de suivi. En observant vous-même le programme à l'action, vous profiterez de l'occasion pour collecter des données qui vous donneront une autre perspective de la situation.

Souvent, l'information la plus utile sur le fonctionnement d'un programme et les améliorations possibles émane des questions qui jettent la lumière sur les faiblesses ou les défauts du programme. Par exemple : 
"Qu'est-ce qui te plaît le moins dans ce projet ? ", ou bien " Comment pourrions-nous améliorer ce programme ? " Les réponses des participantes apportent généralement l'information la plus utile aux réorientations ou modifications futures. Beaucoup de filles hésitent cependant à se montrer critiques ou à exprimer des sentiments négatifs et il existe une certaine tendance à donner la priorité à la politesse, selon un processus que les chercheurs qualifient de "biais de politesse ". Il est ainsi parfois difficile d'obtenir de réponses critiques de la part des bénéficiaires d'un programme. On recherchera alors d'autres façons de poser la question, pour éviter l'impression de négativité. On pourra par exemple sonder : "Tu as dit aimer tout dans ce programme. Si tu énumérais les aspects du programme en ordre de préférence, lequel mettrais-tu en haut de liste ? Et lequel en bas de liste ? Pourquoi ?"

Nous recommandons, dans la mesure du possible, de prendre note des réponses verbatim, plutôt que de les résumer lors de l'entrevue. Les réponses verbatim apportent une information beaucoup plus nuancée et utile aux programmes. Les listes de points résumés rendent plus difficile le recueil de l'information utile exprimée par les répondantes. En résumant les réponses, l'intervieweuse risque d'omettre une information importante pour les gestionnaires. Les entretiens verbatim peuvent aussi être enregistrés sur cassette, pour être écoutés ensuite par les gestionnaires. Veillez dans ce cas à inclure dans la procédure de consentement une demande d'autorisation de l'enregistrement.

\section{Prendre du recul}

Le suivi du programme sur le terrain, y compris les rapides évaluations de suivi décrites plus haut, sont essentielles si l'on veut bien comprendre comment le programme fonctionne et comment il peut être amélioré.

\section{ÉTUDE DE CAS : Suivi qualitatif en vue de l'amélioration du programme}

Biruh Tesfa ("Avenir prometteur " en amharique) est un programme pour filles établi en Éthiopie pour mobiliser et favoriser la rencontre des filles extrêmement pauvres et déscolarisées, sous la conduite d'une femme mentor adulte. Tous les six mois, les gestionnaires du programme recourent à un outil similaire à l'Outil 3.1, pour suivre les perceptions des filles et l'expérience acquise des mentors. À chaque passage, entre 10 et 20 filles sont interviewées, de même que cinq à 10 mentors. Les gestionnaires de Biruh Tesfa profitent de l'occasion pour explorer de nouveaux domaines d'expansion ou changements programmatiques. Ils modifient aussi les questions pour obtenir différents types d'information. Lors d'une série d'entretiens, ils ont ainsi ajouté la question suivante à celles posées aux mentors :

QUESTION : "Que pensez-vous de votre expérience avec les filles handicapées du programme ? " Toutes similaires, les réponses des mentors ont révélé les défis auxquels se trouvent confrontées les filles handicapées. L'une des mentors de Biruh Tesfa a donné cette réponse :

RÉPONSE : " J'ai une fois inscrit une fille qui avait eu la polio. Ses parents étaient très pauvres. Elle n'avait ni fauteuil roulant, ni béquilles... Après avoir assisté aux activités du programme pendant quelques jours, elle n'est plus venue et ses parents ont dit qu'elle ne pouvait pas s'y rendre seule. " (Mentor d'Addis Abeba)

Les gestionnaires de Biruh Tesfa ont ainsi compris la nécessité de consacrer plus d'attention à l'amélioration de l'accès et de la participation des filles handicapées. Des rampes ont été construites aux lieux de rencontre, pour les rendre plus accessibles. Des fonds ont été affectés au transport en taxi ou à l'accompagnement des filles handicapées. Un nouveau partenariat a été formé avec une organisation locale pour handicapés, de manière à inclure des mentors handicapées et à intensifier le recrutement et le soutien au sein de cette population. 
L'examen du programme dans son ensemble, au moyen de méthodes quantitatives, est également source d'information importante. II est utile de suivre le nombre de participantes, leur profil démographique, les tendances de participation ou les éléments programmatiques reçus.

Il est vivement conseillé d'enregistrer individuellement les participantes et les détails les concernant, plutôt que d'estimer leur nombre total, comme le font beaucoup de programmes de jeunesse. L'enregistrement individuel permet aux gestionnaires d'analyser les caractéristiques des participantes et les tendances de participation parmi les différents groupes de membres.

Les Outils 3.2 et 3.3 offrent deux types d'instruments programmatiques utiles au suivi des membres. L'Outil 3.2 est un formulaire d'enregistrement ou d'inscription. Son format permet la collecte d'information concernant chaque fille au moment de son inscription au programme. Le formulaire n'est rempli qu'une fois, à l'inscription. II permet aux gestionnaires de suivre le nombre et le profil des participantes. Dans le contexte clinique, cette approche serait comparable à la fiche remplie lors de la première consultation.

L'Outil 3.3 est un formulaire d'activité. Les activités ou prestations reçues par chaque fille (telle qu'enregistrée sous l'Outil 3.2) dans le cadre du programme, à chaque visite, contact ou réunion de groupe y sont enregistrées. Cet outil permet au personnel et aux gestionnaires du programme de suivre leurs propres activités, y compris les sujets couverts, les activités et les orientations recommandées aux filles. II permet aussi aux gestionnaires de détecter les biais éventuels de couverture des sujets avec différents types de bénéficiaires. Dans le contexte clinique, ce format s'assimile à celui du registre de l'infirmière, dans lequel elle enregistre toutes les clientes consultées et décrit le diagnostic posé et le traitement prescrit à chaque visite. Cet outil est aussi similaire à un journal, reflet des activités journalières du programme.

Le personnel du programme - qu'il s'agisse de pairs éducatrices, de leaders de groupe ou de mentors - doit être invité à remplir ces formulaires en double. Ils sont en effet utiles, au niveau du personnel de terrain, au suivi des activités mêmes du programme et du nombre de filles atteintes. Au niveau de la gestion, ces données peuvent être enregistrées et analysées pour examiner les performances du programme et les tendances d'utilisation. À tous les niveaux, ces outils ont pour objectif d'améliorer le programme et pas d'en juger les résultats.

\section{Analyse des données aux fins de la gestion programmatique}

Les données, ou statistiques de service du programme sont généralement sous-utilisées. Elles peuvent être incluses dans les rapports aux donateurs ou aux autorités supérieures, mais leur pleine valeur est souvent négligée. Il est recommandé aux gestionnaires de développer une culture d'utilisation des données aux fins du suivi et de la gestion de leurs programmes. L'examen de simples indicateurs peut apporter une information précieuse sur les performances du programme (voir l'encadré "Indicateurs utiles au suivi des programmes").

Les Figures 7 à 9 illustrent la manière dont de simples fréquences et tableaux croisés des données peuvent révéler une information précieuse sur les programmes. Les données présentées ici reposent sur les données statistiques de service effectives de programmes proposés aux filles des milieux ruraux d'Éthiopie.
Nous

recommandons une combinaison de stratégies de suivi qui recueillent l'information à la base tout en examinant aussi les résultats au niveau global. 


\section{INDICATEURS UTILES AU SUIVI DES PROGRAMMES}

\section{Indicateurs de performance}

Nombre de filles recrutées par mois

Nombre total de filles recrutées

Nombre de filles qui quittent le programme

Nombre de filles handicapées recrutées

Nombre de filles recrutées par personnel de terrain

Nombre de filles ayant assisté à au mois $X$ sessions du programme

\section{Indicateurs démographiques}

Âges des filles inscrites au programme

Situation scolaire : pourcentage de filles scolarisées et déscolarisées

Niveau de scolarité : Pourcentage de répartition des années de scolarité

Pourcentage des filles vivant sans leurs parents, avec un parent ou avec les deux

Pourcentage des filles actuellement mariées ou l'ayant été

\section{Indicateurs d'utilisation}

Pourcentage des filles recevant une information par thème

Pourcentage des filles recevant une information par thème et état matrimonial

Pourcentage des filles recevant une information par thème et état de handicap

La Figure 7 illustre deux modes de présentation distincts des données de participation ou d'inscription à un programme. Les données sont extraites de l'Outil 3.2, Formulaire d'inscription. Dans le Graphique A, le nombre de filles inscrites à chaque mois d'opération du programme est présenté, mois par mois. Cette présentation permet aux responsables de la mise en œuvre et de la gestion de suivre les performances mensuelles du programme, ainsi que leurs tendances. Par exemple, les pics enregistrés dans le graphique $A$, tous les trois à cinq mois, reflètent peut-être la formation de nouveaux groupes de filles. Les creux reflètent peut-être les périodes de groupes en cours, durant lesquelles l'effort de recrutement est moindre.

Le Graphique B présente les mêmes données, mais sous forme cumulative. Ce format est excellent pour le suivi du progrès vers un objectif ou une cible. Dans cet exemple, nous indiquons l'objectif de 50 mille filles

FIGURE 7 Nombre de filles inscrites dans les groupes de filles mariées en Éthiopie rurale, par mois et cumulatif

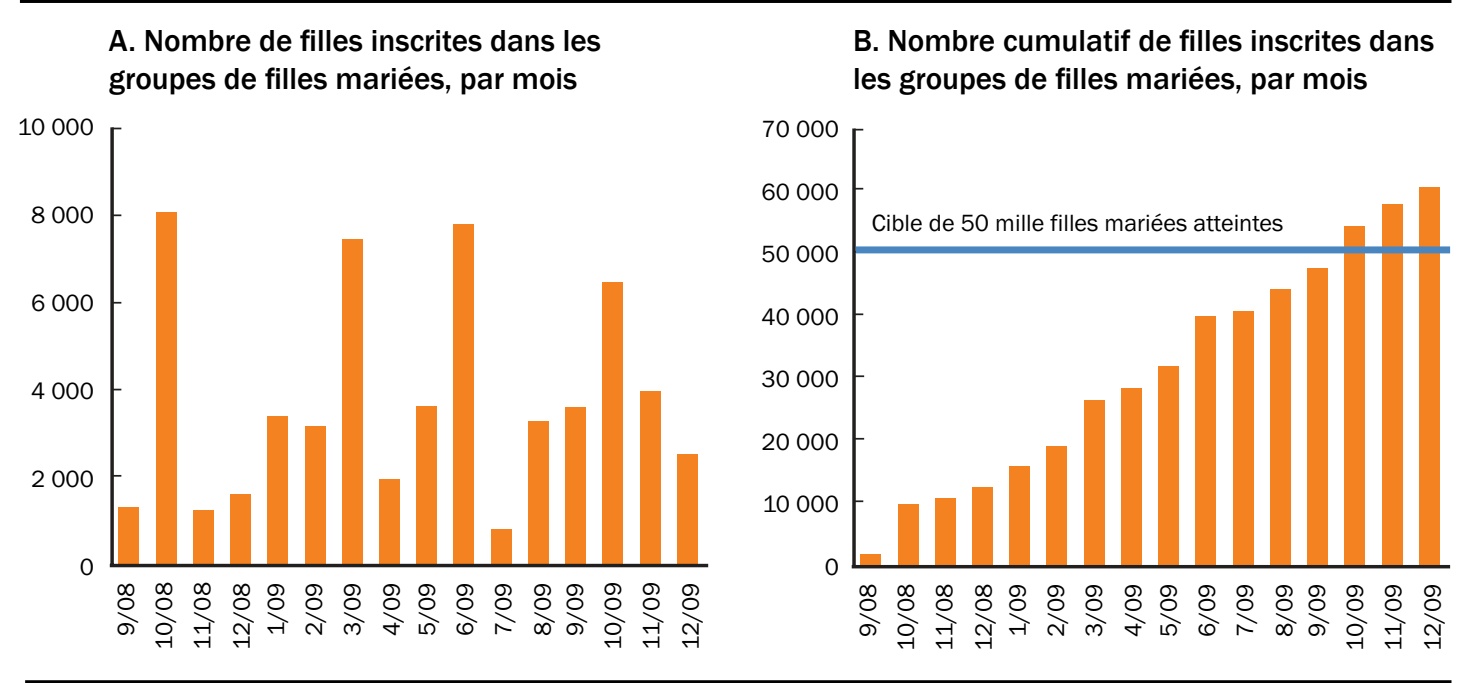


mariées. En actualisant ce type de graphique sur base mensuelle, les gestionnaires peuvent suivre de près la réalisation de leurs cibles et apporter les corrections nécessaires si la progression est faible.

La Figure 8 approfondit l'analyse des données d'iscription en examinant le profil des participantes aux groupes de filles mariées. La grande majorité de ces filles n'a jamais été scolarisée et, parmi celles qui l'ont été, la plupart n'ont atteint que quelques années d'instruction primaire (Graphique A). Cette information n'est pas sans implications en ce qui concerne le contenu du programme. On pourrait par exemple considérer l'ajout d'un enseignement élémentaire. II conviendra aussi de veiller à ce que les activités du programme ne requièrent ni lecture, ni écriture.

Le Graphique B indique la répartition en fonction de l'âge des filles mariées inscrites au programme. La distribution est asymétrique du côté des filles plus âgées, en particulier au-delà de l'âge de 20 ans. Les gestionnaires peuvent en déduire la nécessité d'un plus grand effort de recrutement des filles mariées plus jeunes. Les mentors des groupes de filles mariées doivent aussi veiller à obtenir une déclaration d'âge exacte de la part de leurs membres, certaines jeunes filles mariées hésitant parfois à se révéler mineures.

La Figure 9 approfondit l'analyse de l'information reçue des membres des groupes de filles mariées, en fonction de la tranche d'âge. Les données sont extraites du formulaire d'Activités (Outil 3.3). Elles indiquent que, au moment du rapport de l'information, moins du tiers des filles mariées avaient reçu une information sur le préservatif ou les autres méthodes de planification familiale. Ces données laissent aussi entendre un biais intense contre l'apport de cette information aux plus jeunes filles mariées. Si environ un tiers des aînées ont reçu une information sur la planification familiale, rares sont celles qui en ont bénéficié dans le groupe des cadettes. II en ressort la nécessité pour les gestionnaires de combattre activement les biais et préjugés inconscients que les leaders de groupe ou les mentors peuvent avoir concernant les différents âges des filles mariées. Ces données sont extrêmement utiles au suivi du biais des prestataires et des tendances à éviter certains types de contenu qu'ils jugent peut-être trop sensibles.

FIGURE 8 Âge et profil scolaire des membres de groupes de filles mariées, Éthiopie

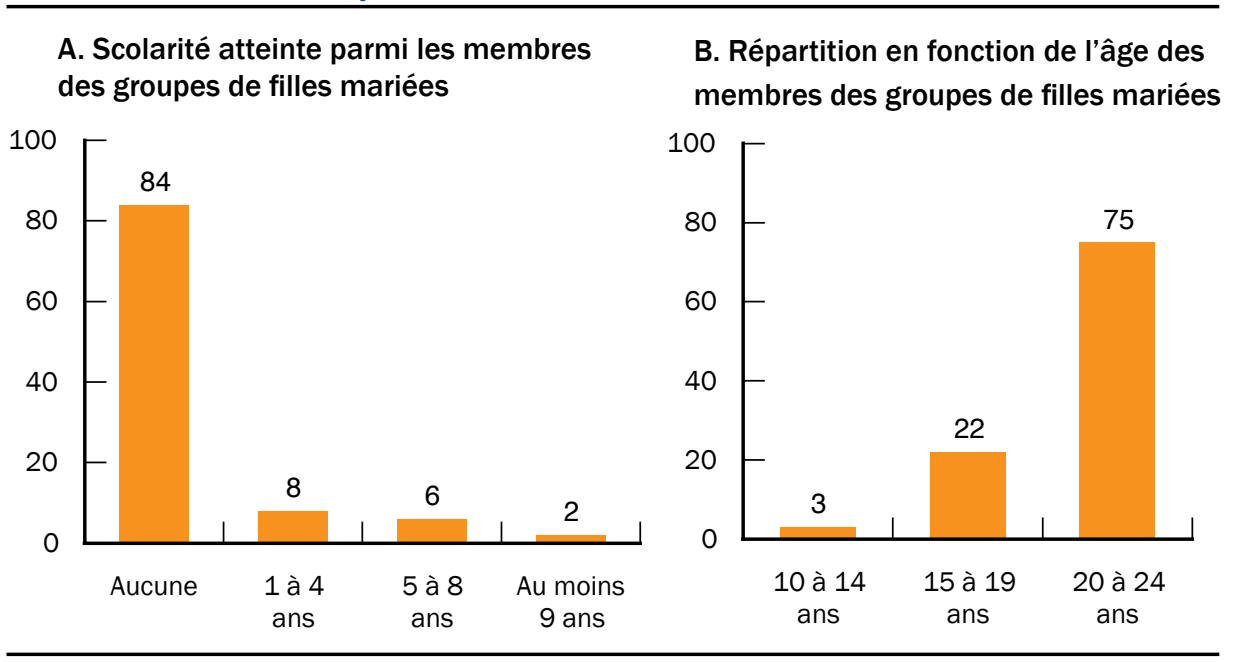


FIGURE 9 Pourcentage des filles mariées ayant reçu une information sur des sujets particuliers, par tranche d'âge
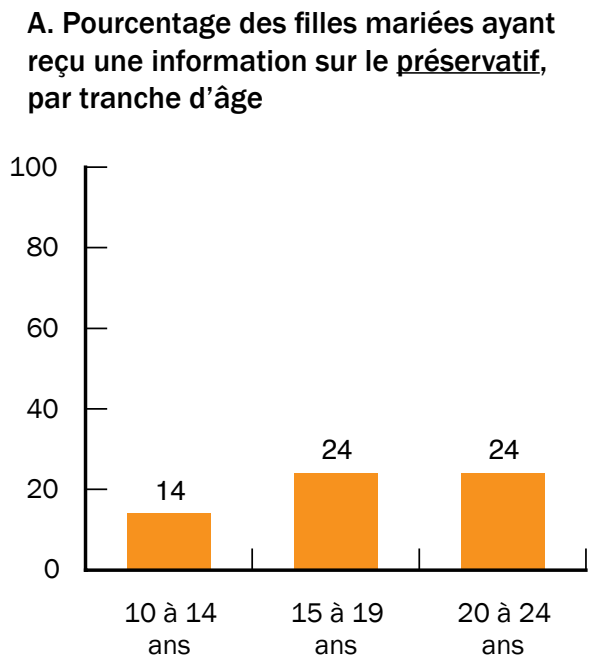

B. Pourcentage des filles mariées ayant reçu une information sur la planification familiale, par tranche d'âge

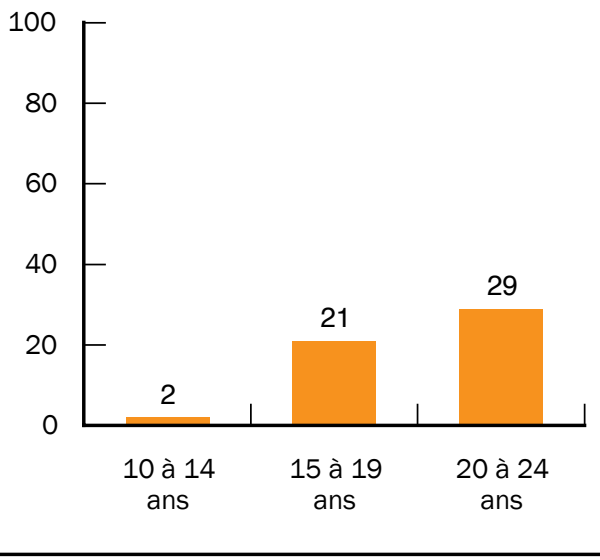

recommandé aux gestionnaires de développer une culture d'utilisation des données aux fins du suivi et de la gestion de leurs programmes.

\section{Remarque concernant la qualité des données statistiques de service}

Les données statistiques de service sont enregistrées par les prestataires: infirmières, pairs éducatrices ou leaders de groupe. Ce type de personnel est rarement formé ou expérimenté en ce qui concerne les formulaires ou questionnaires à remplir. II convient dès lors d'assurer la formation initiale et continue des prestataires à ce sujet; de fréquentes formations de recyclage sont nécessaires aussi. Très souvent, les données collectées auprès des prestataires de service sont de mauvaise qualité, présentent une information incomplète ou non conforme aux systèmes d'encodage requis. L'organisation de formations de recyclage fréquentes est certes recommandée. II n'en demeure pas moins que les données même incomplètes ou imparfaites peuvent être utiles à la gestion des programmes et à leur suivi.

\section{Remarque finale sur les programmes factuels}

Une culture d'utilisation des données aux fins de la conception et du suivi programmatique doit être valorisée et privilégiée dans les programmes destinés aux filles en particulier et aux jeunes en général. II convient de démystifier les méthodes de recherche et l'usage des données et de les rendre pratiques et accessibles aux gestionnaires et au personnel des programmes. Plutôt qu'abstraites, les données générées doivent être concrètes, prêtes à l'emploi, et fournir une information suffisamment détaillée pour donner une orientation pratique, inspirée de sens commun, aux programmes destinés aux filles des milieux ruraux. Le meilleur moyen de privilégier cette culture d'utilisation des données est de faire voir aux gestionnaires et au personnel des programme la valeur inhérente des données et l'avantage qu'elles apportent au programme et aux filles que nous soutenons en Éthiopie. 


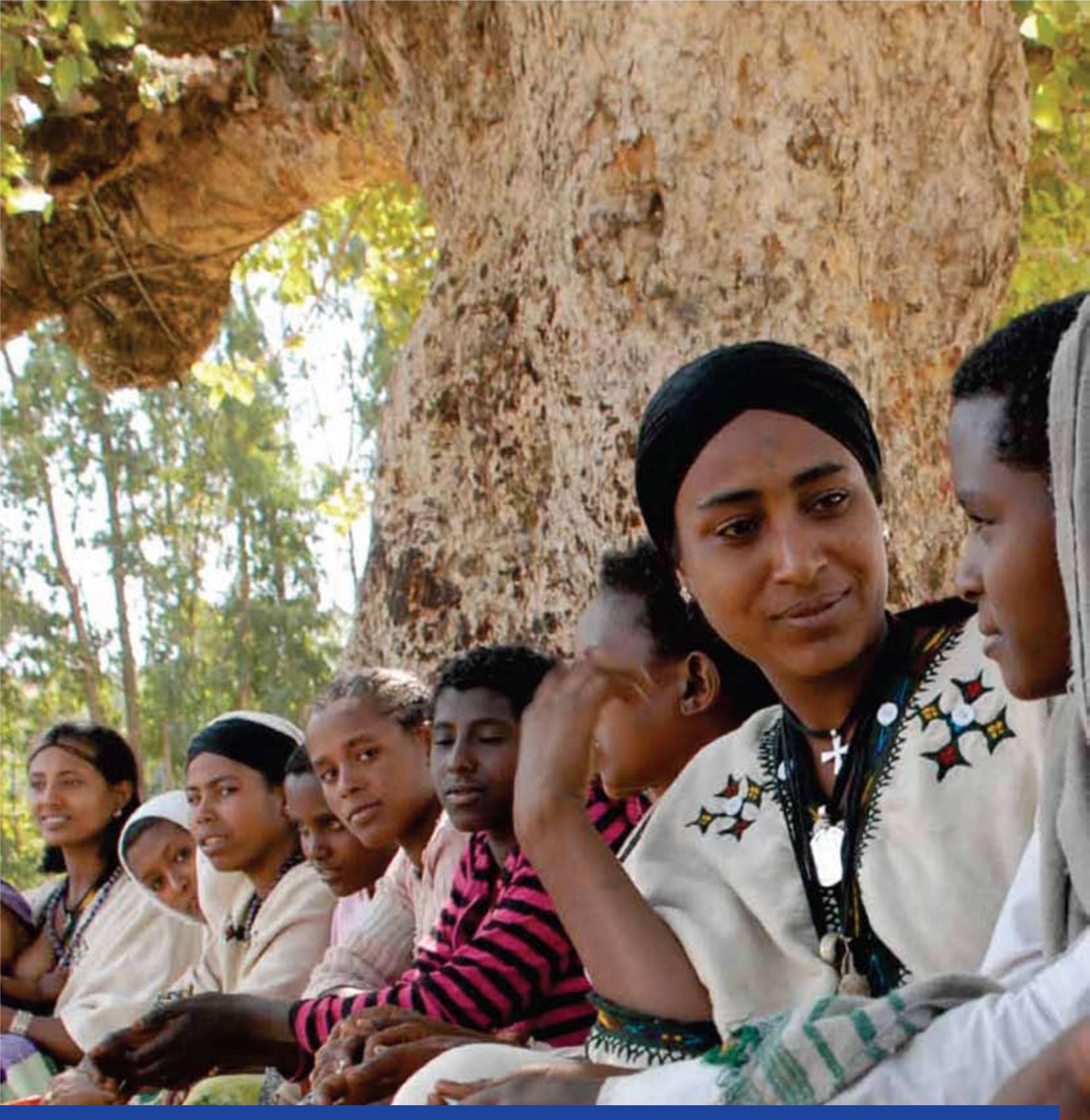

\section{RESSOURCES}

À LA DISPOSITION DES PROGRAMMES DESTINÉS AUX FILLES

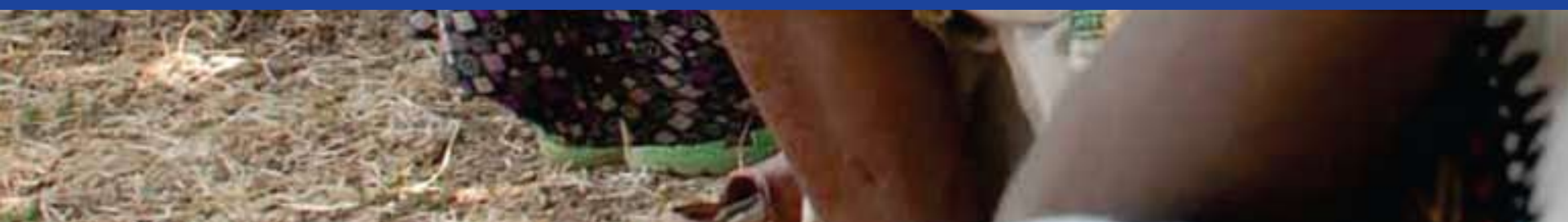





\section{RESSOURCES \\ À LA DISPOSITION DES PROGRAMMES DESTINÉS AUX FILLES}

\section{Méthodes de recherche}

Researching Violence against Women: A Practical Guide for Researchers and Activists ; PATH, 2005. 259 pages. Guide destiné à la planification de la recherche sur les violences faites aux femmes dans les pays en développement, couvrant les complexités d'éthique et les instruments utiles à la collecte de données quantitatives et qualitatives.

http://www.path.org/publications/files/GBV rvaw complete.pdf [en anglais]

\section{Méthodes qualitatives}

Exploring and Understanding Gender in Education: A Qualitative Research Manual for Education Practitioners and Gender Focal Points; UNESCO Bangkok, 2005.

133 pages. Guide de recherche qualitative pour la promotion de l'égalité de genre dans l'enseignement.

http://www.ungei.org/resources/files/Exploring_and_understanding_gender_in_ education UNESCO.pdf [en anglais]

Qualitative Research for Improved Health Programs: A guide to Manuals for Qualitative and Participatory Research on Child Health, Nutrition and Reproductive Health; Academy for Educational Development (AED), 2004. 194 pages. Manuel à l'intention des gestionnaires de programme et des chercheurs dans les domaines sanitaires spécifiques du paludisme, de l'assainissement des eaux, de la nutrition, etc., pour les aider dans la conduite d'une recherche qualitative.

http://sara.aed.org/publications/cross cutting/qualitative/qualitative.pdf [en anglais]

Qualitative Research Methods: A Data Collector's Field Guide; Family Health International, 2005. 136 pages. Guide de collecte de données couvrant l'observation des participants, les entretiens, les groupes de discussion et la gestion des données.

http://www.fhi.org/NR/rdonlyres/emgox4xpcoyrysqspsgy5ww6ma7v4e44 etd6toiejyxalhbmk5sdnef7falr3q6h/wa 2ttj5524xbn/datacollectorguideenrh.pdf [en anglais]

\section{Méthodes quantitatives}

Designing HIV Intervention Studies: An Operations Research Handbook; Population Council, 2002. 156 pages. Guide publié à l'intention des chercheurs sur le VIH pour les aider à rédiger leurs propositions de recherche opérationnelle, avec présentation de certains concepts opérationnels clés.

http://www.popcouncil.org/pdfs/horizons/orhivaidshndbk.pdf [en anglais]

Family Planning Operations Research: A Book of Readings; Population Council, 1999. 389 pages. Recueil d'information sur les programmes de planification familiale et leur recours à la recherche opérationnelle.

http://www.popcouncil.org/pdfs/frontiers/Capacity Bldg/FPORReadings.pdf [en anglais]

Framework for Operations and Implementation Research in Health and Disease Control Programs; The Global Fund, 2009. 72 pages. Ressource primaire pour la planification d'une recherche opérationnelle avec étapes de planification, mise en œuvre et suivi.

http://www.theglobalfund.org/documents/me/FrameworkForOperationsResearch.pdf [en anglais] 


\section{Éthique}

Ethical Approaches to Gathering Information from Children and Adolescents in International Settings: Guidelines and Resources; Horizons, 2005. 73 pages. Principales questions d'éthique et recommandations pratiques d'éthique dans la recherche ayant trait aux enfants et aux jeunes.

http://www.popcouncil.org/pdfs/horizons/childrenethics.pdf [en anglais]

Research Ethics Training Curriculum, Second Edition; Family Health International, 2009. 444 pages. Programme destiné aux chercheurs internationaux, guide de principes d'éthique et études de cas.

http://www.fhi.org/training/en/RETC2/index.html [en anglais]

Cours de formation sur l'éthique de la recherche destiné aux représentants communautaires; Family Health International, 2005. 203 pages. Guide destiné aux représentants communautaires, sur l'importance de l'éthique de la recherche. http://www.fhi360.org/fr/RH/Training/trainmat/ethicscurr/RETCCRFr/index.htm

\section{Programmes d'enseignement et boîtes à outils}

\section{Programmes d'enseignement}

Choose a Future: Issues and Options for Adolescent Girls in India; CEDPA, 2003. 304 pages. Guide destiné aux animateurs chargés d'instruire les adolescentes sur divers sujets, tels que la santé génésique, les objectifs personnels, l'environnement, le droit reconnu par la loi, etc. http://www.cedpa.org [en anglais]

Un SEUL programme : Guides et activités pour une approche pédagogique unifiée de la sexualité, du genre, du VIH et des droits humains; Population Council, 2010. 2 volumes, 187 pages et 280 pages. Manuel destiné à faciliter l'instruction des adolescents sur les questions de santé sexuelle et génésique. Deux volumes, y compris un guide et des activités. Accessible en français sur: http://www.popcouncil.org/publications/books/2010 ItsAllOne.asp.

Life Planning Skills: A Curriculum for Young People in Africa; PATH, 2003. 371 pages. Manuel destiné à faciliter l'instruction des jeunes sur la manière de relever les défis de la vie tels que le $\mathrm{VIH}$, la drogue, la sexualité, etc.

http://www.path.org/files/HIV-TB aya Ips facilitator bots.pdf [en anglais]

Sakhi Saheli - Promoting Gender Equity and Empowering Young Women: A Training Manual; Population Council, 2008. 136 pages. Manuel destiné à l'enseignement des jeunes femmes sur leur vulnérabilité au VIH/sida moyennant une approche axée sur la résolution des normes de genre. http://www.popcouncil.org/pdfs/horizons/India SakhiSaheli Eng.pdf [en anglais]

Working with Young Women: Empowerment Rights and Health; Promundo, 2009. 144 pages. Guide destiné aux animateurs de programmes pour jeunes femmes de 15 à 24 ans, sur les inégalités de genre.

http://www.promundo.org.br/wp-content/uploads/2010/03/trabalhandocom-mulheres-jovens-ingles.pdf [disponible en anglais et en portugais]

\section{Boîtes à outils}

Boîte à outils pour la santé sexuelle et reproductive des adolescents en situations de crise humanitaire; UNFPA, 2009. 94 pages. Boîte à outils créée à l'intention des responsables programmatiques cherchant à évaluer si les programmes de santé génésique répondent adéquatement aux besoins des jeunes. Version française sur http://unfpa.org/webdav/site/global/shared/documents/ publications/2010/UNFPA ASRHtoolkit french.pdf 
Conception d'un programme centré sur les filles : Boîte à outils pour l'élaboration, le renforcement et l'expansion de programmes destinés aux adolescentes; Population Council, 2010. 231 pages. Boîte à outils conçue pour instruire les animatrices sur la manière de travailler avec les adolescentes, couvrant les sujets à aborder et la façon de suivre et évaluer les résultats des programmes. Version française sur http://www.popcouncil.org/pdfs/2011PGY_AdolGirlToolkit_fr.pdf

Investing When It Counts: Generating the Evidence Base for Policies and Programs for Very Young Adolescents ;

Population Council, 2006. 50 pages. Ressource axée sur l'apport de données factuelles au soutien des programmes et politiques visant à atteindre les très jeunes adolescents (10 à 14 ans).

http://www.popcouncil.org/pdfs/InvestingWhenltCounts.pdf [disponible en anglais et en espagnol]

\section{Ressources sur la situation des filles}

The Coalition for Adolescent Girls: Poverty Ends with Her. Fondée par la Fondation des Nations Unies et la Nike Foundation, la Coalition a été rejointe par plus de 30 éminentes organisations internationales, y compris ses membres fondateurs : l'International Center for Research on Women (ICRW), le Population Council et I'International Women's Health Coalition (IWHC). www.coalitionforadolescentgirls.org [en anglais]

Girls Speak: A New Voice in Global Development; Nike Foundation, 2009. 43 pages. Rapport à l'intention des responsables programmatiques, des décideurs politiques et des donateurs sur l'importance de l'investissement dans les fillettes et les filles.

http://www.nikefoundation.org [en anglais]

New Lessons: The Power of Educating Adolescent Girls; Population Council, 2009. 162 pages. Rapport sur l'importance de l'éducation des adolescentes et stratégies de réponse à leurs besoins.

http://www.popcouncil.org/pdfs/2009PGY NewLessons.pdf [en anglais]

Start with a Girl: A New Agenda for Global Health; Nike Foundation, 2009. 96 pages. Guide-programme d'action soulignant l'importance de la santé publique au profit des filles.

http://www.nikefoundation.org [en anglais]

The Unchartered Passage: Girls' Adolescence in the Developing World; Population Council, 1998. 115 pages. Pour comprendre la situation et les circonstances des adolescentes dans les pays en développement et la nécessité de programmes conçus à leur intention. http://www.popcouncil.org [en anglais] 


\section{CONSENTEMENT}

Bonjour. Je m'appelle Je travaille pour [NOM DE VOTRE ORGANISATION] et nous réalisons une étude sur les filles en Éthiopie. Nous aimerions te parler de ta vie dans cette communauté et dans ta famille/ton foyer.

Si tu veux bien participer à l'étude, j'aurai un entretien personnel avec toi pendant lequel je te poserai des questions sur toi-même et sur ta vie, tes opinions et ce que tu fais. Nous n'allons pas te demander beaucoup de temps. L'entretien prendra environ une demi-heure. Tu pourras l'arrêter à tout moment ou choisir de sauter une question si tu préfères ne pas y répondre.

L'information que tu me donneras nous aidera à établir des programmes et améliorer ceux qui existent déjà ici. Si tu acceptes de participer, un risque est que certaines des questions que je te poserai te paraissent très personnelles ou gênantes. D'un autre côté, l'avantage est que tu apporteras une information utile aux responsables de la santé publique, pour qu'ils puissent mieux organiser les programmes destinés aux filles d'Éthiopie.

Ce que tu penses et que tu vis est important pour nous. II faut que tu nous donnes des réponses franches et vraies. Certaines questions te paraîtront peut-être indiscrètes, personnelles et peut-être même déplaisantes. Ta participation est totalement volontaire. Tu peux me demander d'arrêter la discussion si tu ne te sens pas à l'aise, ou tu peux refuser de répondre à une question particulière. Pour les questions déplaisantes auxquelles tu voudras bien répondre, je te demande de répondre en toute honnêteté, parce qu'il est important que je reçoive des réponses complètes et franches.

Les entretiens et les discussions sont strictement confidentiels. Personne ne sera donc mis au courant de tes réponses. Ton nom ne figurera pas sur ce questionnaire, pas plus que sur aucune de mes notes ni dans aucun des rapports préparés. Si tu as des questions, je serai prête à y répondre. Si je ne dispose pas de l'information que tu me demandes, je te le dirai et si tu le désires, j'essaierai d'obtenir la réponse.

Je dois d'abord te poser une ou deux questions à propos de ton âge :

\section{As-tu moins de 18 ans?}

2. Habites-tu avec un parent ou un tuteur?

3. Es-tu d'accord que je demande à ton parent ou tuteur la permission que tu participes à cette étude?
西

$\square \quad$ Non (PASSER À Q5)

4. Si oui, tes parents ou ton tuteur ne seront pas informés des résultats de ton questionnaire. Tes réponses resteront totalement confidentielles et ton parent ou tuteur devra lire/se faire lire et signer ceci :

Je soussigné suis le parent ou tuteur de la personne invitée à participer à l'étude. J'ai lu la formule de consentement ou elle m'a été lue, l'occasion m'a été donnée de clarifier mes questions éventuelles concernant l'étude et je donne la permission à mon enfant ou enfant en tutelle de participer à l'étude.

Signature Date

5. Si non, te paraît-il nécessaire de ne pas informer tes parents ou ton tuteur pour ta propre protection?

OUi (PASSER À Q6)

$\square \quad$ Non (FIN)

6. As-tu des questions ? (PRENDRE NOTE DES QUESTIONS)

Oui

$\square \quad$ Non

7. Es-tu d'accord de participer à l'étude ?

Oui

$\square \quad$ Non

Si tu as des doutes ou des questions dans le futur, tu pourras joindre le responsable de l'étude par téléphone au [NUMÉRO DE TÉLÉPHONE].

Je soussignée, intervieweuse, ai expliqué les procédures de l'étude et ses risques et avantages à la répondante dans une langue qu'elle comprend et elle comprend ces procédures et risques et avantages. 


\begin{tabular}{|c|c|c|}
\hline $\begin{array}{l}\text { Question } \\
n^{\circ}\end{array}$ & Question & $\begin{array}{l}\text { Réponse ou codes de réponse } \\
\text { (Ne pas lire. Remplir la réponse ou entourer le code approprié.) }\end{array}$ \\
\hline \multicolumn{3}{|c|}{ SECTION 1 : INFORMATION DE BASE } \\
\hline 101 & Quel âge as-tu? & I______ ÂGE DE LA RÉPONDANTE \\
\hline 102 & $\begin{array}{l}\text { Avec qui habites-tu : avec ta mère et ton père, } \\
\text { avec ta mère seule, avec ton père seul ou sans } \\
\text { tes parents? }\end{array}$ & $\begin{array}{l}1=\text { AVEC MÈRE ET PÈRE } \\
2=\text { AVEC MĖRE SEULE } \\
3=\text { AVEC PĖRE SEUL } \\
4=\text { SANS PARENT, NI MÈRE NI PÈRE }\end{array}$ \\
\hline 103 & $\begin{array}{l}\text { Je voudrais parler de tes parents (biologiques). } \\
\text { Sont-ils tous les deux en vie? Ta mère seule } \\
\text { est-elle en vie, ton père seul, ou bien sont-ils } \\
\text { tous les deux décédés? }\end{array}$ & $\begin{array}{l}1 \text { = MÈRE ET PÈRE EN VIE } \\
2=\text { MÈRE SEULE EN VIE } \\
3=\text { PĖRE SEUL EN VIE } \\
4=\text { NI MĖRE NI PĖRE EN VIE }\end{array}$ \\
\hline 104 & $\begin{array}{l}\text { Je voudrais parler de ton chef de famille. } \\
\text { Quelle est la relation de ton chef de famille par } \\
\text { rapport à toi ? }\end{array}$ & $\begin{array}{l}1 \text { = PÈRE } \\
2=\text { MĖRE } \\
3=\text { GRAND-PÈRE OU GRAND-MĖRE } \\
4=\text { AUTRE PARENTÉ (ONCLE, TANTE, SCEUR, FRÈRE) } \\
5=\text { MARI } \\
6=\text { BEAU-PÈRE OU BELLE-MÈRE } \\
7=\text { AUTRE PARENT DU MARI } \\
8=\text { EMPLOYEUR } \\
9=\text { RÉPONDANTE ELLE-MÊME } \\
10=\text { AUTRE (PRÉCISER) }\end{array}$ \\
\hline 105 & As-tu un handicap? & $\begin{array}{l}0=\text { NO (PASSER À Q107) } \\
1=\text { OUI }\end{array}$ \\
\hline 106 & Quel type de handicap as-tu? & $\begin{array}{l}1=\text { PHYSIQUE } \\
2=\text { OUIIE/AUDITIF } \\
3=\text { VUE/VISUEL } \\
4=\text { INTELLECTUEL } \\
5=\text { MENTAL } \\
6=\text { AUTRE (PRÉCISER) }\end{array}$ \\
\hline 107 & As-tu un extrait d'acte de naissance? & $\begin{array}{l}0=\text { NON } \\
1=\text { OUI }\end{array}$ \\
\hline 108 & $\begin{array}{l}\text { As-tu une pièce d'identité, comme une carte } \\
\text { d'identité officielle avec ton nom et ta photo? }\end{array}$ & $\begin{array}{l}0=\text { NON } \\
1=\text { OUI }\end{array}$ \\
\hline \multicolumn{3}{|c|}{ SECTION 2 : SCOLARISATION ET MOYENS DE SUBSISTANCE } \\
\hline 201 & As-tu jamais été à l'école? & $\begin{array}{l}0=\text { NON } \\
1=\text { OUI (PASSER À Q203) }\end{array}$ \\
\hline 202 & $\begin{array}{l}\text { Pourquoi n'as-tu jamais été à l'école? } \\
\text { (PLUS D'UNE RÉPONSE POSSIBLE. ENTOURER } \\
\text { TOUTES CELLES APPLICABLES.) } \\
\text { PASSER À Q207 }\end{array}$ & $\begin{array}{l}1=\text { MANQUE DE RESSOURCES FAMILIALES / PAUVRETÉ } \\
2=\text { MARIÉE } \\
3=\text { TROP DE TÂCHES DOMESTIQUES } \\
4=\text { ÉCOLE TROP ÉLOIGNÉE / AUCUNE ÉCOLE DANS LE VOISINAGE } \\
5=\text { PARENTS OPPOSÉS } / \text { NE VOIENT PAS L'UTILITÉ } \\
6=\text { DÉCĖS/SÉPARATION/MALADIE DES PARENTS } \\
7=\text { PAS DE PLACE DISPONIBLE À L'ÉCOLE } \\
8=\text { PAS INTÉRESSÉE } \\
9=\text { MALADIE OU HANDICAP } \\
10=\text { AUTRE (PRÉCISER) }\end{array}$ \\
\hline 203 & À quel âge as-tu commencé l'école? & I______ ÂGE DE DÉBUT DE SCOLARISATION \\
\hline 204 & Combien d'années d'école as-tu achevées? & I_____ ANNÉES DE SCOLARITÉ \\
\hline 205 & Vas-tu actuellement à l'école? & $\begin{array}{l}0=\text { NON } \\
1=\text { OUI (PASSER À Q207) }\end{array}$ \\
\hline 206 & $\begin{array}{l}\text { Pourquoi ne vas-tu plus à l'école? } \\
\text { (PLUS D'UNE RÉPONSE POSSIBLE. ENTOURER } \\
\text { TOUTES CELLES APPLICABLES.) }\end{array}$ & $\begin{array}{l}1=\text { MANQUE DE RESSOURCES FAMILIALES } / \text { PAUVRETÉ } \\
2=\text { MARIÉE } \\
3=\text { TROP DE TÂCHES DOMESTIQUES } \\
4=\text { ÉCOLE TROP ÉLOIGNÉE } / \text { AUCUNE ÉCOLE DANS LE VOISINAGE } \\
5=\text { PARENTS OPPOSÉS } / \text { NE VOIENT PAS L'UTILITÉ } \\
6=\text { DÉCĖS/SÉPARATION/MALADIE DES PARENTS } \\
7 \text { = PAS DE PLACE DISPONIBLE À L'ÉCOLE } \\
8=\text { PAS INTÉRESSÉE } \\
9=\text { MALADIE OU HANDICAP } \\
10=\text { AUTRE (PRÉCISER) }\end{array}$ \\
\hline 207 & $\begin{array}{l}\text { As-tu jamais travaillé pour un salaire, en argent } \\
\text { ou en nature? }\end{array}$ & $\begin{array}{l}0=\text { NON (PASSER À Q212) } \\
1=\text { OUI }\end{array}$ \\
\hline 208 & $\begin{array}{l}\text { Travailles-tu actuellement pour un salaire, en } \\
\text { argent ou en nature? }\end{array}$ & $\begin{array}{l}0=\text { NON } \\
1=\text { OUI }\end{array}$ \\
\hline 209 & $\begin{array}{l}\text { Quel est ton travail actuel ou le travail le plus } \\
\text { récent pour lequel tu as été payée? }\end{array}$ & I__ I TYPE DE TRAVAIL \\
\hline 210 & $\begin{array}{l}\text { Pendant ta dernière semaine de travail, environ } \\
\text { combien d'heures as-tu travaillé à cette activité? }\end{array}$ & I_____ NOMBRE D'HEURES \\
\hline
\end{tabular}




\begin{tabular}{|c|c|c|}
\hline $\begin{array}{l}\text { Question } \\
\mathrm{n}^{\circ}\end{array}$ & Question & $\begin{array}{l}\text { Réponse ou codes de réponse } \\
\text { (Ne pas lire. Remplir la réponse ou entourer le code approprié.) }\end{array}$ \\
\hline 211 & $\begin{array}{l}\text { Pendant ta dernière semaine de travail, environ } \\
\text { combien as-tu gagné pour ton travail payé ? } \\
\text { (SI PAIEMENT EN NATURE, DEMANDER À LA } \\
\text { RÉPONDANTE D'ESTIMER LA VALEUR DU PAIEMENT } \\
\text { EN NATURE REÇU.) }\end{array}$ & $\mid$ \\
\hline 212 & $\begin{array}{l}\text { Pendant ta dernière semaine de travail, environ } \\
\text { combien d'heures as-tu travaillé à des activités } \\
\text { non payées comme les travaux domestiques ou } \\
\text { le travail agricole familial non payé? }\end{array}$ & I____ I NOMBRE D'HEURES \\
\hline \multicolumn{3}{|c|}{ SECTION 3 : RÉSEAUX SOCIAUX, PARTICIPATION ET UTILISATION DU TEMPS } \\
\hline 301 & $\begin{array}{l}\text { Combien d'amies proches as-tu dans ta } \\
\text { communauté ou ton voisinage? }\end{array}$ & 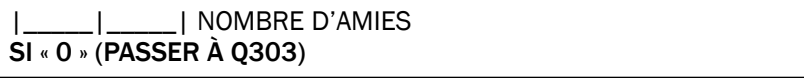 \\
\hline 302 & $\begin{array}{l}\text { As-tu eu des contacts ou as-tu bavardé avec tes } \\
\text { amies pendant cette dernière semaine? }\end{array}$ & $\begin{array}{l}0=\text { NON } \\
1=\text { OUI }\end{array}$ \\
\hline 303 & $\begin{array}{l}\text { Es-tu membre d'un club ou d'un groupe } \\
\text { quelconque dans ta communauté ou ton } \\
\text { voisinage, comme un groupe de jeunes, un } \\
\text { groupe religieux ou un groupe de filles? }\end{array}$ & $\begin{array}{l}0=\text { NON } \\
1=\text { OUI }\end{array}$ \\
\hline 304 & $\begin{array}{l}\text { Je vais te lire une liste de lieux où on peut se } \\
\text { rendre dans la communauté. Je te demande } \\
\text { de me dire si tu t'es rendue dans un de ces } \\
\text { endroits depuis un mois. } \\
\text { (LIRE LA LISTE. ENTOURER TOUTES LES RÉPONSES } \\
\text { APPLICABLES.) }\end{array}$ & $\begin{array}{l}1 \text { = ÉGLISE OU MOSQUÉE } \\
2=\text { GROUPE DE JEUNES/FILLES À L'ÉGLISE OU À LA MOSQUÉE } \\
3=\text { GROUPE DE JEUNES/FILLES EN DEHORS DE L'ÉGLISE OU DE LA } \\
\text { MOSQUÉE } \\
4=\text { CENTRE DE JEUNESSE } \\
5=\text { ÉDUCATION PAR LES PAIRS } \\
6=\text { HÔPITAL PUBLIC OU CENTRE DE SANTÉ } \\
7=\text { CLINIQUE PRIVÉE } \\
8=\text { RÉUNION COMMUNAUTAIRE } \\
9=\text { GROUPE D'ÉPARGNE/ CARROUSEL } \\
10=\text { CONVERSATION COMMUNAUTAIRE }\end{array}$ \\
\hline 305 & $\begin{array}{l}\text { Je voudrais parler du soutien et des réseaux } \\
\text { qui existent peut-être dans ta communauté. } \\
\text { Si tu devais emprunter de l'argent d'urgence, } \\
\text { connais-tu quelqu'un qui pourrait t'en prêter } \\
\text { dans ta communauté? }\end{array}$ & $\begin{array}{l}0=\text { NON } \\
1=\text { OUI }\end{array}$ \\
\hline 306 & $\begin{array}{l}\text { Si tu étais rejetée de chez toi et que tu devais } \\
\text { trouver un endroit où loger, y a-t-il quelqu'un } \\
\text { dans ta communauté qui t'accueillerait? }\end{array}$ & $\begin{array}{l}0=\text { NON } \\
1=\text { OUI }\end{array}$ \\
\hline \multicolumn{3}{|c|}{ SECTION 4 : EXPÉRIENCE SEXUELLE, MARIAGE ET MATERNITÉ } \\
\hline 401 & $\begin{array}{l}\text { As-tu déjà été mariée ou habité avec un } \\
\text { homme comme si tu l'étais? }\end{array}$ & $\begin{array}{l}0=\text { NO (PASSER À Q406) } \\
1=\text { OUI }\end{array}$ \\
\hline 402 & Quel âge avais-tu au moment de ton mariage? & I_____ I ÂGE AU MARIAGE \\
\hline 403 & $\begin{array}{l}\text { Quel âge avait ton mari au moment de votre } \\
\text { mariage? }\end{array}$ & I_____ Â_GE DU MARI AU MARIAGE \\
\hline 404 & $\begin{array}{l}\text { Est-ce que ton mariage a été arrangé par ta } \\
\text { famille ou choisi par toi et ton mari? }\end{array}$ & $\begin{array}{l}1=\text { ARRANGÉ } \\
2=\text { CHOISI }\end{array}$ \\
\hline 405 & $\begin{array}{l}\text { Es-tu actuellement mariée, divorcée, séparée } \\
\text { ou veuve? }\end{array}$ & $\begin{array}{l}1=\text { ACTUELLEMENT MARIÉE } \\
2=\text { DIVORCÉE } / \text { SÉPARÉE } \\
3=\text { VEUVE }\end{array}$ \\
\hline 406 & As-tu déjà eu des rapports sexuels? & $\begin{array}{l}0=\text { NON (FIN D'ENQUÊTE) } \\
1=\text { OUI }\end{array}$ \\
\hline 407 & $\begin{array}{l}\text { À quel âge as-tu eu tes premiers rapports } \\
\text { sexuels? }\end{array}$ & I_____ Â_GE AUX PREMIERS RAPPORTS SEXUELS \\
\hline 408 & $\begin{array}{l}\text { Quel âge avait ton premier partenaire sexuel au } \\
\text { moment de tes premiers rapports? }\end{array}$ & I_____ ÂGE DU PREMIER PARTENAIRE \\
\hline 409 & $\begin{array}{l}\text { Lors de tes premiers rapports sexuels, les } \\
\text { désirais-tu, ne les désirais-tu pas ou n'étais-tu } \\
\text { pas sûre? }\end{array}$ & $\begin{array}{l}1=\text { RAPPORTS SEXUELS DÉSIRÉS } \\
2=\text { RAPPORTS SEXUELS NON DÉSIRÉS } \\
3=\text { PAS SÛRE }\end{array}$ \\
\hline 410 & $\begin{array}{l}\text { Lors de tes premiers rapports sexuels, as-tu été } \\
\text { physiquement forcée ou contrainte à avoir ces } \\
\text { rapports? }\end{array}$ & $\begin{array}{l}0=\text { NON } \\
1=\text { OUI, PHYSIQUEMENT FORCÉE } \\
2=\text { OUI, CONTRAINTE }\end{array}$ \\
\hline 411 & $\begin{array}{l}\text { Lors de tes premiers rapports sexuels, étais-tu } \\
\text { célibataire, fiancée ou mariée? }\end{array}$ & $\begin{array}{l}1=\text { CÉLIBATAIRE } \\
2=\text { FIANCÉE } \\
3=\text { MARIÉE }\end{array}$ \\
\hline
\end{tabular}




\begin{tabular}{|c|c|c|}
\hline $\begin{array}{l}\text { Question } \\
n^{\circ}\end{array}$ & Question & $\begin{array}{l}\text { Réponse ou codes de réponse } \\
\text { (Ne pas lire. Remplir la réponse ou entourer le code approprié.) }\end{array}$ \\
\hline 412 & $\begin{array}{l}\text { De quelles méthodes de planification familiale } \\
\text { as-tu entendu parler? } \\
\text { (LIRE LA LISTE. ENTOURER TOUTES LES RÉPONSES } \\
\text { APPLICABLES.) }\end{array}$ & $\begin{array}{l}1=\text { INJECTABLE } / \text { DEPO } \\
2=\text { PILULE } \\
3=\text { PRÉSERVATIF } \\
4=\text { STÉRILET } \\
5=\text { IMPLANT } \\
6=\text { AUTRE (PRÉCISER) } \\
9=\text { AUCU.................................................. }\end{array}$ \\
\hline 413 & $\begin{array}{l}\text { As-tu déjà utilisé l'injectable, la pilule, } \\
\text { le préservatif ou une autre méthode de } \\
\text { planification familiale? }\end{array}$ & $\begin{array}{l}0=\text { NO (PASSER À Q417) } \\
1=\text { OUI }\end{array}$ \\
\hline 414 & $\begin{array}{l}\text { Quelle méthode de planification familiale as-tu } \\
\text { déjà utilisée? } \\
\text { (LIRE LA LISTE. ENTOURER TOUTES LES RÉPONSES } \\
\text { APPLICABLES.) }\end{array}$ & $\begin{array}{l}1=\text { INJECTABLE } / \text { DEPO } \\
2=\text { PILULE } \\
3=\text { PRÉSERVATIF } \\
4=\text { STÉRILET } \\
5=\text { IMPLANT } \\
6=\text { AUTRE (PRÉCISER) }\end{array}$ \\
\hline 415 & $\begin{array}{l}\text { Utilises-tu actuellement l'injectable, la pilule, } \\
\text { le préservatif ou une autre méthode de } \\
\text { planification familiale? }\end{array}$ & $\begin{array}{l}0=\text { NO (PASSER À Q417) } \\
1=\text { OUI }\end{array}$ \\
\hline 416 & $\begin{array}{l}\text { Quelle méthode de planification familiale } \\
\text { utilises-tu actuellement? } \\
\text { (LIRE LA LISTE. ENTOURER TOUTES LES RÉPONSES } \\
\text { APPLICABLES.) } \\
\text { PASSER À Q418 }\end{array}$ & $\begin{array}{l}1=\text { INJECTABLE } / \text { DEPO } \\
2=\text { PILULE } \\
3=\text { PRÉSERVATIF } \\
4=\text { STÉRILET } \\
5=\text { IMPLANT } \\
6=\text { AUTRE (PRÉCISER) }\end{array}$ \\
\hline 417 & $\begin{array}{l}\text { Pourquoi n'utilises-tu pas de méthode de } \\
\text { planification familiale? } \\
\text { (PLUS D'UNE RÉPONSE POSSIBLE. ENTOURER } \\
\text { TOUTES LES RÉPONSES APPLICABLES.) }\end{array}$ & $\begin{array}{l}1 \text { = DÉSIR DE GROSSESSE } \\
2=\text { ENCEINTE } / \text { ACCOUCHEMENT RÉCENT } \\
3=\text { OPPOSITION DU PARTENAIRE } \\
4=\text { OPPOSITION DE LA RÉPONDANTE } \\
5=\text { IGNORANCE DE LA PLANIFICATION FAMILIALE } \\
6=\text { NE SAIT PAS OŨ OBTENIR LA PLANIFICATION FAMILIALE } \\
7=\text { AUTRE (PRÉCISER) }\end{array}$ \\
\hline 418 & As-tu déjà accouché ? & $\begin{array}{l}0=\text { NON } \\
1=\text { OUI }\end{array}$ \\
\hline 419 & As-tu déjà été enceinte? & $\begin{array}{l}0=\text { NON (FIN D'ENQUÊTE) } \\
1=\text { OUI }\end{array}$ \\
\hline 420 & Es-tu actuellement enceinte? & $\begin{array}{l}0=\text { NON } \\
1=\text { OUI }\end{array}$ \\
\hline 421 & De combien d'enfants as-tu déjà accouché ? & I_____ I NOMBRE D'ENFANTS \\
\hline 422 & $\begin{array}{l}\text { Quel âge avais-tu au moment de ton premier } \\
\text { accouchement? }\end{array}$ & I_____ I ÂGE AU PREMIER ACCOUCHEMENT \\
\hline 423 & $\begin{array}{l}\text { As-tu reçu des soins médicaux ou bénéficié } \\
\text { d'un suivi de santé pendant ta grossesse? }\end{array}$ & $\begin{array}{l}0=\text { NON } \\
1=\text { OUI }\end{array}$ \\
\hline 424 & Où as-tu accouché de ton premier enfant? & $\begin{array}{l}1=\text { CHEZ MOI } \\
2=\text { DANS LA BROUSSE OU DANS LES CHAMPS } \\
3=\grave{A} \text { LA CLINIQUE } \\
4=\grave{A} \text { L'HÔPITAL } \\
5=\text { AUTRE (PRÉCISER) }\end{array}$ \\
\hline 425 & $\begin{array}{l}\text { Qui t'as aidée lors de ton premier } \\
\text { accouchement? }\end{array}$ & $\begin{array}{l}1=\text { PERSONNE } \\
2=\text { MĖRE } / \text { BELLE-MĖRE } \\
3=\text { AUTRE PARENTE } \\
4=\text { ACCOUCHEUSE TRADITIONNELLE } \\
5=\text { AGENT SANITAIRE } \\
6=\text { PERSONNEL MÉDICAL (MÉDECIN, INFIRMIĖRE, AGENT CLINIQUE) } \\
7=\text { AUTRE (PRÉCISER) }\end{array}$ \\
\hline
\end{tabular}

\section{Merci beaucoup de ta participation à cette enquête.}




\section{CONSENTEMENT}

Bonjour. Je m'appelle Je travaille pour [NOM DE VOTRE ORGANISATION] et nous réalisons une étude sur les filles en Éthiopie. Nous aimerions te parler de ta vie dans cette communauté et dans ta famille/ton foyer.

Si tu veux bien participer à l'étude, j'aurai un entretien personnel avec toi pendant lequel je te poserai des questions sur toi-même et sur ta vie, tes opinions et ce que tu fais. Nous n'allons pas te demander beaucoup de temps. L'entretien prendra environ une demi-heure.

L'information que tu me donneras nous aidera à établir des programmes et améliorer ceux qui existent déjà ici. Si tu acceptes de participer, un risque est que certaines des questions que je te poserai te paraissent trop personnelles ou gênantes. D'un autre côté, l'avantage est que tu apporteras une information utile aux responsables de la santé publique, pour qu'ils puissent mieux organiser les programmes destinés aux filles d'Éthiopie.

Ce que tu penses et que tu vis est important pour nous. II faut que tu nous donnes des réponses franches et vraies. Certaines questions te paraîtront peut-être indiscrètes, personnelles et peut-être même déplaisantes. Ta participation est totalement volontaire. Tu peux me demander d'arrêter la discussion si tu ne te sens pas à l'aise, ou tu peux refuser de répondre à une question particulière. Pour les questions déplaisantes auxquelles tu voudras bien répondre, je te demande de répondre en toute honnêteté, parce qu'il est important que je reçoive des réponses complètes et franches.

Les entretiens et les discussions sont strictement confidentiels. Personne ne sera donc mis au courant de tes réponses. Ton nom ne figurera pas sur ce questionnaire, pas plus que sur aucune de mes notes ni dans aucun des rapports préparés. Si tu as des questions, je serai prête à y répondre. Si je ne dispose pas de l'information que tu me demandes, je te le dirai et si tu le désires, j'essaierai d'obtenir la réponse.

Je dois d'abord te poser une ou deux questions à propos de ton âge :

1. As-tu moins de 18 ans?

2. Habites-tu avec un parent ou un tuteur?

3. Es-tu d'accord que je demande à ton parent ou tuteur la permission que tu participes à cette étude?
OUi (PASSER À Q2)

OUi (PASSER À Q3)

OUi (PASSER À Q4) $\square \quad$ Non (PASSER À Q5)

Non (PASSER À Q5)

Non (PASSER À Q6)

4. Si oui, tes parents ou ton tuteur ne seront pas informés des résultats de ton questionnaire. Tes réponses resteront totalement confidentielles et ton parent ou tuteur devra lire/se faire lire et signer ceci :

Je soussigné suis le parent ou tuteur de la personne invitée à participer à l'étude. J'ai lu la formule de consentement ou elle m'a été lue, l'occasion m'a été donnée de clarifier mes questions éventuelles concernant l'étude et je donne la permission à mon enfant ou enfant en tutelle de participer à l'étude.

Signature Date

5. Si non, te paraît-il nécessaire de ne pas informer tes parents ou ton tuteur pour ta propre protection?

$\square \quad$ Oui (PASSER À Q6) $\quad \square \quad$ Non (FIN)

6. As-tu des questions ? (PRENDRE NOTE DES QUESTIONS)

Oui

$\square \quad$ Non

7. Es-tu d'accord de participer à l'étude?

Oui

Non

Si tu as des doutes ou des questions dans le futur, tu pourras joindre le responsable de l'étude par téléphone au [NUMÉRO DE TÉLÉPHONE].

Je soussignée, intervieweuse, ai expliqué les procédures de l'étude et ses risques et avantages à la répondante dans une langue qu'elle comprend et elle comprend ces procédures et risques et avantages. 


\begin{tabular}{|c|c|c|}
\hline \multicolumn{3}{|c|}{ SECTION 1 : INFORMATION DE BASE } \\
\hline 101 & Quel âge as-tu? & I____ I ÂGE DE LA RÉPONDANTE \\
\hline 102 & $\begin{array}{l}\text { Avec qui habites-tu : avec ta mère et ton père, } \\
\text { avec ta mère seule, avec ton père seul ou sans } \\
\text { tes parents? }\end{array}$ & $\begin{array}{l}1=\text { AVEC MÈRE ET PÈRE } \\
2=\text { AVEC MĖRE SEULE } \\
3=\text { AVEC PĖRE SEUL } \\
4=\text { SANS PARENT, NI MÈRE NI PÈRE }\end{array}$ \\
\hline 103 & $\begin{array}{l}\text { Je voudrais parler de tes parents (biologiques). } \\
\text { Sont-ils tous les deux en vie ? Ta mère seule } \\
\text { est-elle en vie, ton père seul, ou bien sont-ils } \\
\text { tous les deux décédés? }\end{array}$ & $\begin{array}{l}1=\text { MÈRE ET PÈRE EN VIE } \\
2=\text { MĖRE SEULE EN VIE } \\
3=\text { PĖRE SEUL EN VIE } \\
4=\text { NI MĖRE NI PĖRE EN VIE }\end{array}$ \\
\hline 104 & As-tu jamais été à l'école? & $\begin{array}{l}0=\text { NON (PASSER À SECTION 2) } \\
1=\text { OUI }\end{array}$ \\
\hline 105 & À quel âge as-tu commencé l'école? & I_____ ÂGE DE DÉBUT DE SCOLARISATION \\
\hline 106 & Combien d'années d'école as-tu achevées? & I______ ANNÉES DE SCOLARITÉ \\
\hline 107 & Vas-tu actuellement à l'école? & $\begin{array}{l}0=\text { NON } \\
1=\text { OUI }\end{array}$ \\
\hline
\end{tabular}

\section{SECTION 2 : HISTOIRE, CONTEXTE FAMILIAL ET COMMUNAUTÉ}

201. Parle-moi de toi. Dis-moi tout ce que tu veux à ton sujet, pour m'aider à te connaître. (Sondes possibles : Où es-tu née ? Comment es-tu arrivée ici ? Que penses-tu de cette communauté ?)

202. Parle-moi de ta famille ou des personnes avec qui tu vis. (Sondes possibles : Avec qui habites-tu ? Comment es-tu arrivée à cet arrangement? Que penses-tu de ta situation familiale/de logement? Qu'est-ce que tu aimes le plus dans ta situation familiale/de logement ? Quels aspects aimerais-tu changer ou améliorer? Pourquoi ?)

203. Parle-moi de ta communauté. (Sondes possibles: Qu'est-ce que tu aimes le plus dans ta communauté ? Qu'est-ce que tu aimes le moins dans ta communauté ? Pourquoi ? Qu'est-ce que tu aimerais changer et pourquoi ?)

204. Je voudrais parler des moments où tu te sens la plus heureuse (ou le plus en sécurité) et de ceux où tu l'es moins. Dis-moi ce qui, dans ta vie, te rend la plus heureuse. Parle-moi des moments où tu te sens le plus en sécurité. (Sondes possibles : Peux-tu me donner un exemple ? Décris-moi la dernière fois où tu t'es sentie extrêmement heureuse/en sécurité. Qu'est-ce qui se passait à ce moment-là ?) 
205. Dis-moi ce qui, dans ta vie, te rend malheureuse. Parle-moi de moments où tu ne t'es pas sentie en sécurité. (Sondes possibles : Peux-tu me donner un exemple ? Décris-moi la dernière fois où tu t'es sentie extrêmement malheureuse/en danger. Qu'est-ce qui se passait à ce moment-là ?)

\section{SECTION 3 : SCOLARISATION}

301. J'aimerais de parler de l'école. Quelle est-ton expérience de l'école ? (Si la répondante n'a jamais été scolarisée, demander pourquoi elle ne va pas à l'école.) (Sondes possibles : Qu'est-ce que tu aimais le plus dans ton école ? Qu'est-ce que tu aimais le moins ? Pourquoi ? Peux-tu me donner un exemple de la dernière fois où tu n'as pas aimé quelque chose ? Comment te rendais-tu à l'école ? Combien de temps te fallait-il pour aller à l'école ?)

302. Parle-moi de tes maîtres d'école. Parle-moi d'un maître ou d'une maîtresse d'école que tu aimais vraiment et de ce que tu appréciais en lui ou en elle. Parle-moi d'un enseignant que tu aimais moins. Qu'est-ce qui te déplaisait en lui ou en elle ? (Sondes possibles: Peux-tu me donner un exemple ? Peux-tu me parler d'une situation où tu n'as vraiment pas aimé cet enseignant?)

303. J'aimerais parler de l'atmosphère qui règne à ton école : en classe, dans la cour de récréation et avec les autres élèves. Quand tu penses à l'atmosphère et aux autres élèves de ton école, qu'est-ce que tu aimes le mieux ? Quand te senstu la plus heureuse ? (Sondes possibles: Qu'est-ce que tu aimes dans les autres élèves ? Qu'est-ce que tu aimes dans la cour de récréation ou l'espace commun? Quelle est ton activité préférée à l'école ?)

304. Quand tu penses à l'atmosphère et aux autres élèves de ton école, qu'est-ce que tu aimes le moins ? Quand te sens-tu malheureuse ou en danger? (Sondes possibles : Est-ce qu'on te taquine ? Parle-moi d'une situation où on t'a taquinée. Te sens-tu parfois menacée ? Parle-moi d'une situation où tu t'es sentie menacée.) 
305. Parle-moi de tes résultats scolaires. (Sondes possibles : Travailles/travaillais-tu bien ou aurais-tu pu faire mieux? Qu'est-ce qui t'a empêchée de faire mieux ? Qu'est-ce qui devrait changer pour que tu puisses faire mieux ? Peux-tu me donner un exemple? Peux-tu me donner un exemple d'une situation où tu n'as pas eu de bons points et pourquoi ?)

306. Parle-moi du moment où tu as quitté l'école ou d'un moment où tu as pensé que tu devrais quitter l'école. As-tu vécu un tel moment ? Quelles étaient les circonstances? (Sondes possibles : Pourquoi as-tu dû quitter l'école ? Qu'est-ce qui a amené la possibilité de devoir quitter l'école ? Qui était impliqué dans la situation ? Et toi, qu'en pensais-tu ?)

307. Aimerais-tu me dire autre chose de ton expérience de l'école ou de ta vie, en général, dont nous n'avons pas parlé ? Parle-moi d'un autre problème ou d'un autre aspect de l'école ou de ta vie qui est important pour toi.

Merci beaucoup de ta participation à cet entretien. 
REMARQUE À LA PERSONNE CHARGÉE DE LA COLLECTE DES DONNÉES : INSÉRER LA FORMULE DE CONSENTEMENT ICI (VOIR L'OUTIL 1.2 POUR EXEMPLE).

\begin{tabular}{|c|c|c|}
\hline \multicolumn{3}{|c|}{ SECTION 1 : INFORMATION DE BASE } \\
\hline 101 & Quel âge as-tu? & I______ ÂGE DE LA RÉPONDANTE \\
\hline 102 & $\begin{array}{l}\text { Avec qui habites-tu : avec ta mère et ton père, } \\
\text { avec ta mère seule, avec ton père seul ou sans } \\
\text { tes parents? }\end{array}$ & $\begin{array}{l}1 \text { = AVEC MÈRE ET PÈRE } \\
2 \text { = AVEC MĖRE SEULE } \\
3 \text { = AVEC PĖRE SEUL } \\
4 \text { = SANS PARENT, NI MÈRE NI PÈRE (PRÉCISER AVEC QUI) }\end{array}$ \\
\hline 103 & $\begin{array}{l}\text { Je voudrais parler de tes parents (biologiques). } \\
\text { Sont-ils tous les deux en vie? Ta mère seule } \\
\text { est-elle en vie, ton père seul, ou bien sont-ils } \\
\text { tous les deux décédés? }\end{array}$ & $\begin{array}{l}1 \text { = MÈRE ET PÈRE EN VIE } \\
2=\text { MÈRE SEULE EN VIE } \\
3=\text { PĖRE SEUL EN VIE } \\
4=\text { NI MĖRE NI PĖRE EN VIE }\end{array}$ \\
\hline 104 & As-tu jamais été à l'école? & $\begin{array}{l}0=\text { NON (PASSER À Q106) } \\
1=\text { OUI }\end{array}$ \\
\hline 105 & Combien d'années d'école as-tu achevées? & I_____ ANNÉES DE SCOLARITÉ \\
\hline 106 & $\begin{array}{l}\text { En quel mois de quelle année t'es-tu inscrite au } \\
\text { programme? }\end{array}$ & I_____ MOIS I___________ I ANNÉE \\
\hline 107 & $\begin{array}{l}\text { À quelle partie du programme participes-tu? } \\
\text { (LIRE LA LISTE. ENTOURER TOUTES LES } \\
\text { RÉPONSES APPLICABLES.) }\end{array}$ & $\begin{array}{l}\text { (ADAPTER CETTE SECTION AU PROGRAMME DE L'ORGANISATION) } \\
1 \text { = SESSIONS DE MENTORING } \\
2 \text { = CONVERSATIONS COMMUNAUTAIRES } \\
3 \text { = GROUPES D'ENTRAIDE } \\
4 \text { = ENSEIGNEMENT INDIVIDUEL } \\
5=\text { AUTRE (PRÉCISER) }\end{array}$ \\
\hline
\end{tabular}

\section{SECTION 2 : EXPÉRIENCE DU PROGRAMME}

201. Où/comment as-tu appris l'existence du programme [NOM DU PROGRAMME] ? (Sondes possibles : Quelle était ton impression à son sujet? Comment les autres en parlaient-ils?)

202. Parle-moi de ta participation au programme. À quelles activités participes-tu ? (Sondes possibles : Te rends-tu souvent aux activités du programme? Qu'as-tu appris dans ce programme ? Que penses-tu du programme ?)

203. Parle-moi [DE TON MENTOR / DE TA PAIR ÉDUCATRICE / DU LEADER DE TON GROUPE / DES AUTRES PERSONNES RESPONSABLES DU PROJET]. (Sondes possibles : Quel est son(leur) rôle dans le programme ? Que penses-tu de cette(ces) personne(s)? Est-ce qu'elle(s) t'a(ont) aidée ?) 
204. Je voudrais parler de l'horaire et du lieu du programme. Est-ce que ces aspects du programme te conviennent ou y changerais-tu quelque chose si tu le pouvais ? Que penses-tu de l'espace de rencontre ? Dis-moi ce que tu changerais si tu le pouvais. (Sondes possibles : Aimerais-tu changer certaines choses ? Qu'aimerais-tu voir changer ? Comment ?)

205. Que pensent tes parents/tuteurs du programme ? Qu'en disent-ils ? Que disent les gens de ta communauté du programme ? (Sondes possibles : Y a-t-il eu un moment où ils ne voulaient pas que tu ailles au programme ? Pourquoi ? Raconte-moi ce qui s'est passé.)

\section{SECTION 3 : PERCEPTIONS ET SUGGESTIONS D'AMÉLIORATION}

301. Je voudrais te demander de réfléchir aux différents aspects du programme [NOM DU PROGRAMME] : ce que tu apprends, les activités auxquelles tu participes, ton ou ta [MENTOR / PAIR ÉDUCATRICE / LEADER DE GROUPE / AUTRE PERSONNEL RESPONSABLE DU PROJET] et ton interaction avec les autres filles. Qu'est-ce que tu aimes le plus dans le programme ? (Sondes possibles : Pourquoi est-ce ton aspect préféré ? Peux-tu me donner un exemple ? Parle-moi de la session du programme qui t'a le plus plu. Que s'est-il passé à cette session?)

302. Réfléchis encore aux différents aspects du programme. Qu'est-ce que tu aimes le moins ? Qu'est-ce que tu aimerais améliorer? (Sondes possibles : Pourquoi est-ce l'aspect que tu aimes le moins ? Peux-tu me donner un exemple? Parlemoi d'une fois où le programme ne t'a pas tant plu et où tu aurais aimé y changer quelque chose. Que s'est-il passé à cette session?)

303. Sur quels sujets ou dans quels domaines aimerais-tu recevoir plus d'information ? Quel type d'information aimerais-tu recevoir? (Sondes possibles : Pourquoi penses-tu que tu as besoin de plus d'information? Peux-tu me donner un exemple? Et en ce qui concerne les compétences ? Y a-t-il d'autres compétences dont tu penses avoir besoin ? Dis-moi pourquoi ces compétences sont importantes pour toi.) 
304. Peux-tu me donner quelques idées sur la manière dont nous pourrions améliorer le programme ? Quels changements ou améliorations aimerais-tu voir? (Sondes possibles : Quelle autre information aimerais-tu recevoir ? Quelles activités voudrais-tu voir proposées ? Comment changerais-tu la façon d'agir [DE TON MENTOR / DE TA PAIR ÉDUCATRICE / DU LEADER DE TON GROUPE / DES AUTRES PERSONNES RESPONSABLES DU PROJET] ?)

305. J'aimerais que tu réfléchisses maintenant aux autres filles de ta communauté. Y a-t-il dans ta communauté des filles qui aimeraient se joindre au programme mais qui ne le font pas ? Quelles sont les raisons pour lesquelles elles ne s'inscrivent pas? (Sondes possibles: Peux-tu me donner un exemple de fille qui aimerait venir mais qui ne peut pas ? Parlemoi de sa situation et de la raison pour laquelle elle ne vient pas.)

306. Y a-t-il d'autres choses que tu penses que je devrais savoir à propos du programme, pour que nous puissions l'améliorer pour toi et pour les autres filles de la communauté? N'hésite pas à me dire tout ce que tu voudrais que je sache.

Merci beaucoup de ta participation à cet entretien. 
OUTIL 3.2

FORMULAIRE D'INSCRIPTION À UN PROGRAMME DESTINÉ AUX FILLES

RÉGION

ZONE

WOREDA

QEBELÉ

MENTOR

MOIS

ANNÉE

À remplir par les mentors au moment de l'inscription des filles.

Remplir en double exemplaire et remettre la copie à la responsable lors des réunions mensuelles.

\begin{tabular}{|c|c|c|c|c|c|c|c|c|c|c|c|}
\hline 1 & 2 & 3 & 4 & 5 & 6 & 7 & 8 & 9 & 10 & 11 & 12 \\
\hline 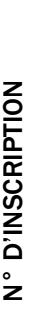 & | & 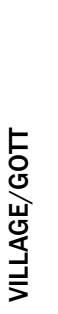 & $\begin{array}{l}\text { NOM } \\
\text { (FACULTATIF) }\end{array}$ & 岁 & $\begin{array}{l}\text { SITUATION } \\
\text { SCOLAIRE } \\
\text { 1=SCOLARISÉE } \\
\text { 2=DÉSCOLARI- } \\
\quad \text { SÉE }\end{array}$ & $\begin{array}{l}\text { ANNÉES DE } \\
\text { SCOLARITÉ }\end{array}$ & 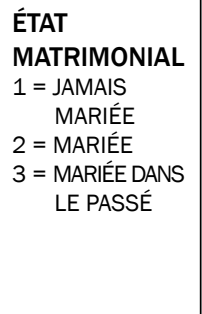 & $\begin{array}{l}\text { MIGRANTE } \\
0=\text { NON } \\
1=\mathrm{OUI}\end{array}$ & $\begin{array}{l}\text { HANDI- } \\
\text { CAPÉE } \\
0=\text { NON } \\
1=\text { OUI }\end{array}$ & $\begin{array}{l}\text { OCCUPA- } \\
\text { TION } \\
\text { (DÉCRIRE) }\end{array}$ & $\begin{array}{l}\text { AVEC QUI } \\
\text { HABITES- } \\
\text { TU ? } \\
1=\text { DEUX } \\
\text { PARENTS } \\
2=U N \\
\text { PARENT } \\
3=\text { SANS } \\
\text { PARENT } \\
\text { (SPÉCIFIER) }\end{array}$ \\
\hline 04 & 14 & o & $\begin{array}{l}\text { Genet } \\
\text { Demele }\end{array}$ & 16 & 2 & 4 & 2 & 1 & 0 & $\begin{array}{l}\text { Ménagère/ } \\
\text { Travaux } \\
\text { agricoles }\end{array}$ & 3 (mari) \\
\hline & & & & & & & & & & & \\
\hline & & & & & & & & & & & \\
\hline & & & & & & & & & & & \\
\hline & & & & & & & & & & & \\
\hline & & & & & & & & & & & \\
\hline & & & & & & & & & & & \\
\hline & & & & & & & & & & & \\
\hline & & & & & & & & & & & \\
\hline & & & & & & & & & & & \\
\hline & & & & & & & & & & & \\
\hline & & & & & & & & & & & \\
\hline & & & & & & & & & & & \\
\hline & & & & & & & & & & & \\
\hline & & & & & & & & & & & \\
\hline & & & & & & & & & & & \\
\hline & & & & & & & & & & & \\
\hline & & & & & & & & & & & \\
\hline & & & & & & & & & & & \\
\hline & & & & & & & & & & & \\
\hline & & & & & & & & & & & \\
\hline & & & & & & & & & & & \\
\hline
\end{tabular}


OUTIL 3.3

FORMULAIRE D'ACTIVITÉS DE PROGRAMME DESTINÉ AUX FILLES

RÉGION

ZONE

WOREDA

QEBELÉ

MENTOR

MOIS

ANNÉE

À remplir par les mentors à chaque réunion, activité ou rencontre avec les filles.

Remplir en double exemplaire et remettre la copie à la responsable lors des réunions mensuelles.

\begin{tabular}{|c|c|c|c|c|c|c|c|c|c|c|c|}
\hline 1 & 2 & 3 & 4 & 5 & 6 & 7 & 8 & 9 & 10 & 11 & 12 \\
\hline 놀 & MOIS & ANNÉE & $\begin{array}{c}\text { NOM } \\
\text { (FACULTATIF) }\end{array}$ & ÂGE & \multicolumn{3}{|c|}{$\begin{array}{l}\text { SUJETS COUVERTS } \\
\text { (3 CODES MAX) }\end{array}$} & \multicolumn{3}{|c|}{$\begin{array}{c}\text { ACTIVITÉS } \\
\text { (3 CODES MAX) }\end{array}$} & $\begin{array}{l}\text { ORIENTATION } \\
\text { VERS UN AUTRE }\end{array}$ \\
\hline 04 & Mars & 2003 & $\begin{array}{c}\text { Genet } \\
\text { Demele }\end{array}$ & 16 & 2 & 4 & $\stackrel{8}{M G F}$ & 1 & 3 & & (CDV) \\
\hline & & & & & & & & & & & \\
\hline & & & & & & & & & & & \\
\hline & & & & & & & & & & & \\
\hline & & & & & & & & & & & \\
\hline & & & & & & & & & & & \\
\hline & & & & & & & & & & & \\
\hline & & & & & & & & & & & \\
\hline & & & & & & & & & & & \\
\hline & & & & & & & & & & & \\
\hline & & & & & & & & & & & \\
\hline & & & & & & & & & & & \\
\hline & & & & & & & & & & & \\
\hline & & & & & & & & & & & \\
\hline & & & & & & & & & & & \\
\hline & & & & & & & & & & & \\
\hline & & & & & & & & & & & \\
\hline & & & & & & & & & & & \\
\hline & & & & & & & & & & & \\
\hline
\end{tabular}

\section{CODES DE SUJETS}

$1=\mathrm{VIH} / \mathrm{SIDA}$

$2=$ PLANIFICATION FAMILIALE

$3=$ SANTÉ ET HYGIËNE

$4=$ GROSSESSE ET MATERNITÉ
$5=$ VIOLENCE DE GENRE

$6=$ HANDICAPS

$7=$ COMMUNICATION

8 = AUTRE (PRÉCISER)

\section{CODES D'ACTIVITÉS}

$1=$ LEÇON AU GROUPE

2 = DISCUSSION DE GROUPE

$3=$ MISE EN SCÈNE/JEU DE RÔLE

$4=$ SPORTS
$5=$ DANSE

$6=$ ART ET ARTISANAT

$7=$ CONFÉRENCE

$8=$ AUTRE (PRÉCISER) 